\title{
A case study of Nairobi City Council's decentralised syphilis screening programme in antenatal clinics
}

Baker Ndugga Maggwa

Ian Askew

Population Council

Elizabeth Mugwe

Bilhah Hagembe

Rick Homan

Follow this and additional works at: https://knowledgecommons.popcouncil.org/departments_sbsr-rh

Part of the Demography, Population, and Ecology Commons, International Public Health Commons, and the Maternal and Child Health Commons How does access to this work benefit you? Let us know!

\section{Recommended Citation}

Maggwa, Baker Ndugga, lan Askew, Elizabeth Mugwe, Bilhah Hagembe, and Rick Homan. 2001. "A case study of Nairobi City Council's decentralised syphilis screening programme in antenatal clinics," FRONTIERS Report. Nairobi: Population Council. 


\section{A Case Study of Nairobi City Council's Decentralised Syphilis Screening Programme in Antenatal Clinics}

1 Population Counal POPULATION COUNCIL

Baker Ndugga Maggwa

Ian Askew

Elizabeth Mugwe

NAIROBI CITY COUNCIL

Bilhah Hagembe

FAMILY HEALTH INTERNATIONAL Rick Homan 


\section{A Case Study of Nairobi City Council's Decentralised Syphilis Screening Programme in Antenatal Clinics}

Population Council

Baker Ndugga Maggwa

Ian Askew

Elizabeth Mugwe

Nairobi City Council

Bilhah Hagembe

Family Health International

Rick Homan

\section{January 2001}

This study was funded by the Joint United Nations Programme on HIV/AIDS (UNAIDS) under the terms of Technical Services Agreement HQ/99/467251, and the U.S. Agency for International Development (USAID) under the terms of Cooperative Agreement number HRN-A-00-98-00012-00. The opinions expressed herein are those of the authors and do not necessarily reflect the view of UNAIDS or USAID. 


\section{SUMMARY}

Syphilis sero-reactivity rates of three to six percent have been reported for many years in Nairobi, as in many other parts of sub-Saharan Africa. It has long been known that syphilis is one of the more serious sexually transmitted infections (STI), especially during pregnancy when, if untreated, at least 60 percent of infected women will experience an adverse pregnancy outcome. There has been renewed interest in its control and prevention because of its proven link with HIV transmission.

In 1989, as part of the establishment of a National STD Control Programme within the Kenyan Ministry of Health $(\mathrm{MOH})$, integration of routine syphilis screening and management into antenatal care was made one of the highest priorities. In 1992 the Nairobi City Council (NCC) pilot-tested a decentralized approach to syphilis screening and management in a sample of their antenatal clinics. This project was supported by the MotherCare Project of John Snow Inc., the University of Nairobi, the Institute of Tropical Medicine (Belgium), the University of Ghent (Belgium), the University of Manitoba (Canada), and the Health and AIDS Unit of the European Commission. The decentralized model differed from the existing centralized approach in four crucial aspects: on-site testing of women by clinic staff; use of the Rapid Plasma Reagin (RPR) test; treatment of RPR sero-reactive women at the antenatal clinics by nurses; and active promotion of partner notification and presumptive treatment of partners of positive women.

This case study was carried out to assess the effectiveness, readiness and cost of the NCC's antenatal care programme, with a particular focus on the decentralized syphilis screening and treatment service. The study collected and compared data from eleven clinics using the decentralized model and seven standard clinics. Data were collected through client-provider observations, client exit interviews, focus group discussions and in-depth interviews with providers, a patient-flow analysis and a cost analysis.

The findings reveal that the proportion of antenatal clients who were screened for maternal syphilis increased from 62 percent in standard clinics using off-site testing, to 100 percent when the NCC first adopted the decentralized approach. Over time, however, the proportion of clients tested has declined to 81 percent in the pilot clinics using the decentralized approach, and to 51 percent in the standard clinics with off-site testing. This decline is attributed primarily to the frequent shortages of test kits, needles and syringes, a situation exacerbated by the recent shift in financial support from external donors to the NCC itself.

There has also been a sizeable increase in the proportion of antenatal clients testing positive who are treated. Prior to decentralization, only nine percent of positive clients were treated, and this increased to 87 percent immediately after the pilot project and has increased to 95 percent currently. Moreover, the proportion of positive clients attending the standard clinics who are treated has also increased to 95 percent, because they are referred to the pilot clinics for both testing and treatment. This finding suggests that, in urban areas where clinics are in close proximity, treatment need not necessarily be available at every facility.

Partner notification and treatment have also improved. Following the pilot study, 50 percent of partners of positive women were treated, and this rate has risen more recently to approximately 70 percent. This is probably due to active promotion of the importance of partner notification when counselling positive women, and to the use of a notification slip. 
However, despite this improved effectiveness, the NCC health facilities overall are not well prepared to provide good quality antenatal services, including syphilis screening and management. There are no specific guidelines or appropriate job-aids for antenatal services and a significant proportion of health facilities lack the essential equipment, supplies and medications required. Many health facilities do not have appropriate IEC materials and clinic staff rarely give group health talks. There is also a high turnover of trained staff, with the result that staff who are inadequately prepared and trained are providing services.

The quality of antenatal care at the pilot clinics is no better than, and in some aspects is worse than, that in the standard clinics. Clients attending both types of clinics are rarely assessed for clinical evidence of syphilis infection and most clients are not educated and/or counselled about maternal syphilis, other STIs, HIV/AIDS and other common reproductive health problems like genital cancer. Infection prevention procedures are not always followed, especially when drawing blood for the syphilis test and most clinics lack appropriate facilities for disposing of sharp instruments. The quantity and quality of supervision have reduced significantly over time, and the quality control procedures that were established are now on the verge of collapse. Clients spend a lot of time waiting for services at the clinics, and new clients spend approximately 20 minutes in consultations. Providers spend a relatively small proportion of their paid time in direct contact with clients.

On average, it costs the NCC US\$5.40 at the pilot clinics and US\$6.60 at the standard clinics per new antenatal client visit without syphilis screening and treatment. This rises to US\$7.60 and US\$11.30 respectively when syphilis screening and treatment are included. Introducing the decentralized approach at the standard clinics would, therefore, increase the per-visit costs from US\$ 6.60 to US\$ 7.60, a difference of only US\$1.00. Given the high prevalence of adverse pregnancy outcomes associated with untreated maternal syphilis, this additional cost to the programme should be justifiable.

In conclusion, decentralization of maternal syphilis screening and management is feasible in a public sector, urban programme and, when implemented properly, leads to more antenatal clients and their partners being screened and treated. In common with most antenatal services in sub-Saharan Africa, however, the NCC clinics are insufficiently prepared to offer good quality antenatal services and to ensure that syphilis screening and treatment are available for all antenatal clients because of poorly trained staff and a lack of basic supplies. 
SUMMARY.

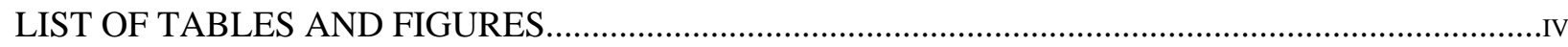

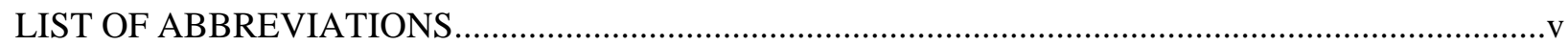

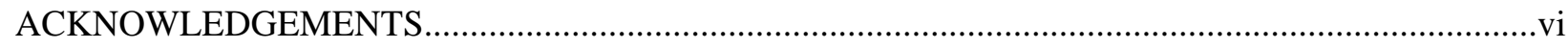

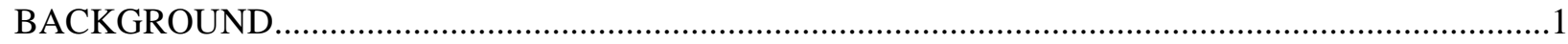

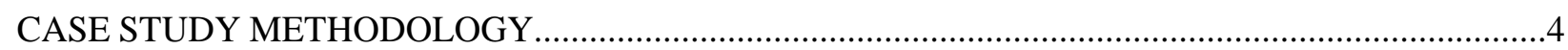

READINESS TO PROVIDE ANTENATAL CARE AND SYPHILIS SCREENING AND TREATMENT

Service Provider Guidelines On Antenatal Care ................................................................

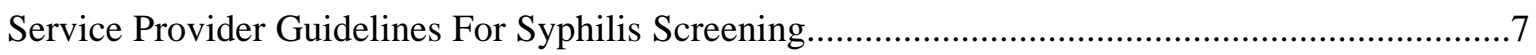

Equipment And Supplies For Providing Antenatal Care ..............................................................

Equipment And Supplies For Syphilis Testing And Management................................................

Information, Education And Communication Activities.................................................................11

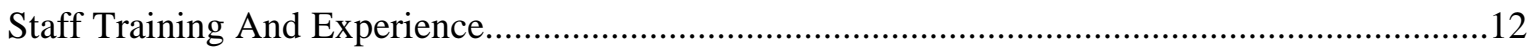

IMPLEMENTATION OF THE DECENTRALISED MODEL

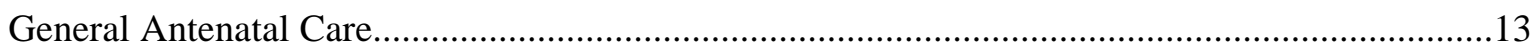

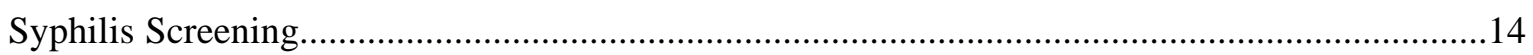

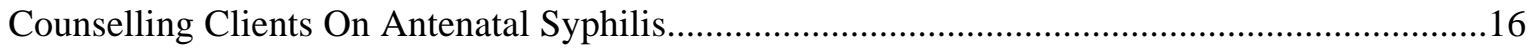

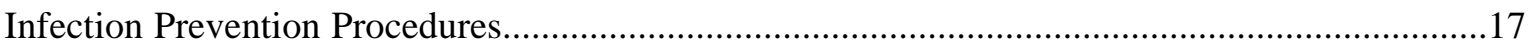

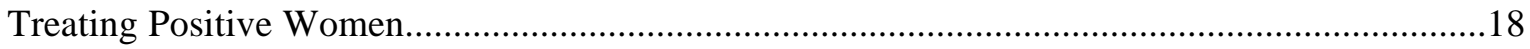

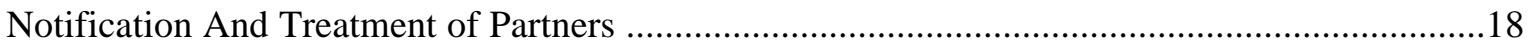

Assessing Clients' For Other Reproductive Tract Problems......................................................19

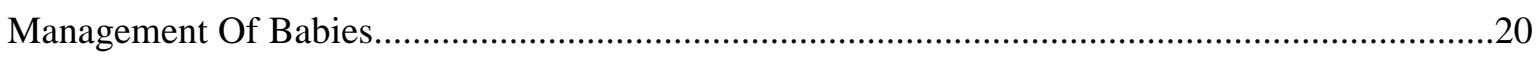

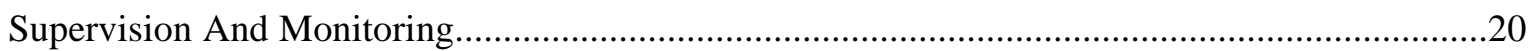

Time Utilization By Clients And Providers At The Clinics.............................................................

PROGRAMME PERFORMANCE.

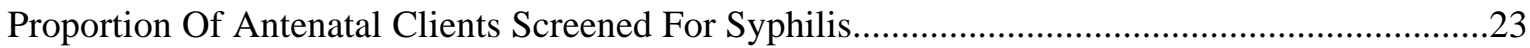

Proportion Of Antenatal Clients Testing Positive That Are Treated..............................................24

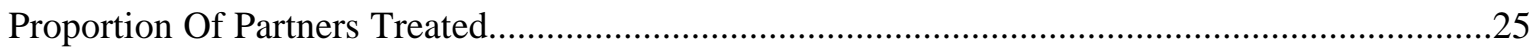

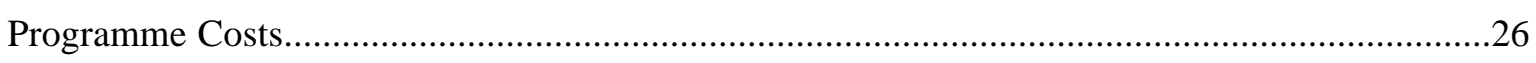

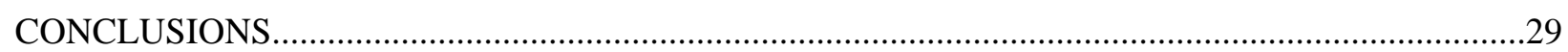

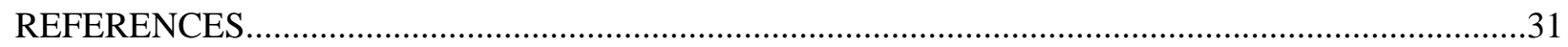

APPENDIX : NCC CLINICS INCLUDED IN THE CASE STUDY …................................................... 


\section{LIST OF TABLES AND FIGURES}

Table 1 Percentage of clinics with essential equipment for antenatal care .8

Table 2 Percentage of clinics with essential equipment and supplies for syphilis screening.. .10

Table 3: Availability of IEC Materials .11

Figure 1: Percentage of clinics with drugs for treating syphilis .11

Figure 2 Percentage of new antenatal clients assessed by condition .......................................13

Figure 3 Percentage of new antenatal clients counselled about pregnancy ...............................14

Figure 4 Percentage of new ANC clients assessed for syphilis by type of assessment .............15

Figure $5 \quad$ Percentage of new antenatal clients informed about syphilis.....................................16

Figure 6 Percentage of cases in which infection prevention procedures were followed during blood drawing.

Figure 7 Percentage of antenatal clients asked about symptoms or examined for clinical signs of reproductive tract problems at pilot and standard clinics.

Figure 8 Time utilization at the clinic

Figure 9: Proportion of antenatal clients screened for syphilis.

Figure 10: Proportion of antenatal clients testing positive who were treated...... .25

Figure 11 Costs per visit by type of client management protocol 27 


\section{LIST OF ABBREVIATIONS}

\begin{tabular}{|c|c|}
\hline $\mathrm{AMOH}$ & Assistant Medical Officers for Health \\
\hline ANC & Antenatal Care \\
\hline CIDA & Canadian International Development Agency \\
\hline DfID & Department for International Development \\
\hline FGDs & Focus Group Discussions \\
\hline FP & Family Planning \\
\hline HIV/AIDS & $\begin{array}{l}\text { Human Immunodeficiency Virus/ Acquired Immune Deficiency } \\
\text { Syndrome }\end{array}$ \\
\hline IEC & Information, Education and Communication \\
\hline KDHS & Kenya Demographic Health Survey \\
\hline $\mathrm{MCH} / \mathrm{FP}$ & Maternal and Child Health/Family Planning \\
\hline $\mathrm{MOH}$ & Kenya Ministry of Health \\
\hline $\mathrm{NCC}$ & Nairobi City Council \\
\hline PFA & Patient Flow Analysis \\
\hline RTI & Reproductive Tract Infection \\
\hline RPR & Rapid Plasma Reagin \\
\hline STC & Special Treatment Clinic \\
\hline STI & Sexually Transmitted Infection \\
\hline STD & Sexually Transmitted Disease \\
\hline TDS & Three times a day \\
\hline TPHA & Treponema Pallidum Heamagglutination Assay \\
\hline UON & University of Nairobi \\
\hline UNAIDS & Joint United Nations Programme on HIV/AIDS \\
\hline USAID & United States Agency for International Development \\
\hline VDRL & Venereal Disease Research Laboratory \\
\hline VCT & Voluntary Counselling and Testing \\
\hline WHO & World Health Organisation \\
\hline
\end{tabular}




\section{ACKNOWLEDGEMENTS}

This study was undertaken with substantial support from many people whose assistance is gratefully acknowledged. At the Nairobi City Council, special thanks go to the Medical Officer of Health, the clinic staff and the clients who agreed to participate in the study and for all the support they gave to the study team. Special thanks also go to Kevin O'Reilly, Bidia Deperthes and Francis Ndowa of WHO and UNAIDS in Geneva for their contribution to the development of the study protocols, review of the draft reports and for finding the financial resources used to carry out the case study. At the Population Council, special thanks go to John Townsend, Monica Wanjiru, Violet Bukusi and Joanne Lewa. 


\section{BACKGROUND}

Several studies in Africa have reported high prevalence rates of syphilis among the general population as well as among pregnant women, ranging from three to sixteen percent. In Kenya, syphilis sero-reactivity rates of approximately three percent have been reported in urban settings for many years (Temmerman et al, 1993, Temmerman et al, 2000). Although syphilis has long been known to be a particularly serious sexually transmitted infection (STI), interest in its control and prevention has been revived in this era of the HIV/AIDS epidemic. This has been due to the recognition that syphilis is one of the STIs that usually presents with a genital ulcer and genital ulcers are known to facilitate HIV transmission.

Syphilis infection during pregnancy can also have severe adverse consequences. If untreated, it is estimated that at least 60 percent of pregnant women with syphilis will experience an adverse pregnancy outcome which include stillbirths, neonatal deaths, premature delivery, and spontaneous abortions. Based on models developed for the assessment of sequelae of maternal syphilis, 20 percent of pregnancies among women infected with syphilis will result in abortions, 30 percent in stillbirths, 25 percent will be born with congenital syphilis, 12.5 percent will be premature births and only 12.5 percent will be healthy infants (van Dam, 1995).

To improve the management of maternal syphilis, most national programmes and donors in Sub-Saharan Africa are shifting their strategies towards a broader reproductive health service approach, which would include integrating STI and HIV/AIDS prevention and treatment services into existing MCH/FP programmes. This shift is based on the belief that integration of these services will lead to more cost-effective and better quality services, because the basic infrastructure and staff that exist for $\mathrm{MCH} / \mathrm{FP}$ services could routinely offer these services with minimal additional inputs, and both sets of services target similar population (i.e. the sexually active). It is under these circumstances that in 1989, integration of routine syphilis screening and management in antenatal clinics was made one of the highest priorities in the establishment of a National STD Control Programme within the Kenyan Ministry of Health $(\mathrm{MOH})$.

\section{Nairobi City Council's Antenatal Syphilis Screening Programme}

The Nairobi City Council (NCC) provides health services to city residents through a network of static and community based programmes. The NCC has 54 health facilities providing reproductive health services in its eight administrative divisions. The vast majority (94\%) of women in Nairobi receive antenatal care from a registered nurse, nurse midwife or doctor at least once during pregnancy (KDHS, 1998). Most of the women who attend antenatal care start before the sixth month of pregnancy and make several visits, with 61 percent attending four or more times.

Prior to 1989, the NCC clinics used to send all their blood specimens to the National Laboratory based at Kenyatta National Hospital for syphilis testing. In 1989, the Council received technical and financial assistance to introduce a system in which blood would be drawn from women at their first antenatal visit and the specimens transported to their own central laboratory at Pumwani Hospital to be tested using the Venereal Disease Research Laboratory (VDRL) or the Treponema Pallidum Haema-glutination Assay (TPHA) tests (Temmerman et al, 1993). Results from the central laboratory were sent back to the referring clinic where the client was informed of the test result. 
This process of taking blood, getting the test done, and feeding the results back to the referring clinics took between two and four weeks. Once the results were available at the clinics, women testing positive for syphilis were then referred to the Nairobi Special Treatment Clinic (STC), the only STD treatment centre in the city at the time.

A review of this centralized process six months from inception of the new strategy (Temmerman et al, 1993) revealed that 61.5 percent of women attending antenatal clinics were tested at their first visit. Of the women whose blood was taken for testing, although most (87 percent) had their test results registered on their antenatal clinic card, thus indicating that the results were being fed back to the clinics, only 9.1 percent of the women testing positive received treatment. The review concluded that the following were some of the reasons for the poor performance of this approach.

- Misplacing specimens during transfer from the clinics to the central laboratory.

- Women did not return for their results because of the long interval between giving the blood specimen and receiving the result. This meant that no treatment was given to these women even when their sero status was known.

- The distance to the only STD treatment centre as well as the stigma attached to it prevented some women from getting treatment even when they knew their test results.

\section{The Decentralised Syphilis Screening Model}

In view of these findings, the NCC decided to pilot-test a decentralized approach to syphilis screening and management in an effort to increase effectiveness. The pilot study was jointly implemented and funded by the NCC, the MotherCare Project of John Snow Inc., the University of Nairobi, the Institute of Tropical Medicine (Belgium), the University of Ghent (Belgium), the University of Manitoba (Canada), and the Health and AIDS Unit of the European Commission. This new model of service delivery, described fully in the accompanying box, differed from the existing approach in four crucial aspects:

- On-site testing of women by clinic staff.

- Use of the Rapid Plasma Reagin (RPR) test.

- Treatment of RPR sero-reactive women at the antenatal clinics by nurses.

- Active promotion of partner notification and presumptive treatment of partners of RPR sero-reactive women.

The main objective of the pilot project was to test the feasibility and cost-effectiveness of this decentralised model. The model was implemented in nine NCC health centres selected on the basis of serving the largest lower income population situated in urban and peri-urban areas. The pilot project was initiated in June 1992 and the counselling component began in October 1992, with staff training being completed by January 1993. The project was evaluated over the period July 1992 to August 1993 (Jenniskens et al, 1995). The evaluation showed that the 
decentralised model had greatly improved the detection and management of antenatal syphilis as follows:

- Virtually all (99.9\%) antenatal clients were now being screened, i.e. having their blood taken and tested.

- Overall, 6.5 percent tested positive (range 2.7 - 9\% across the nine clinics).

- 87 percent of the women testing positive received treatment at the clinics where they had been tested (range $74.6-100 \%$ across the nine clinics).

- 48 percent of the partners of positive clients were also treated at the same clinic as the index antenatal client (range 37.3-72.9\% across the nine clinics).

The results from this pilot study indicated that a decentralised model for on-site diagnosis and treatment of maternal and partner syphilis could be effective. With the demonstrated success of the approach, the NCC increased the number of clinics implementing this model to 14, with additional funding coming from the European Commission and CIDA.

\section{The Nairobi City Council}

\section{Decentralized Maternal Syphilis Screening and Treatment Programme}

Providing laboratory equipment, supplies, drugs and reagents: Syphilis serology was performed by nurses working in the antenatal clinics using the RPR card test (RPR, Wellcome, London, UK). Screening units were set up in the pilot health centres' $\mathrm{MCH}$ clinics and provided with minimal laboratory equipment and supplies, drugs and counselling facilities. Laboratory equipment consisted of a centrifuge and a RPR card shaker. A reference laboratory at the University of Nairobi (UON) performed quality control (Macro-value, RPR card test, Becton Dickinson, Cockeysville, USA). Each MCH clinic stored all serum samples collected during each day of the week for re-testing at the UON laboratory, and the results were discussed with the clinic staff on a monthly basis. RPR sero-reactive women were treated with a single dose of 2.4 million units of intra-muscular benzathine penicillin.

Training of antenatal clinic nurses in performing a rapid syphilis screening test: A manual was developed for training antenatal clinic nurses on the public health importance of maternal syphilis, screening and diagnosis of syphilis in pregnancy, and antenatal syphilis control programmes; components of patient care, RPR testing techniques, promoting behaviour change and counselling women with syphilis and their partners using syphilis counselling materials. Using this training manual a total of 69 antenatal clinic nursing staff in the pilot programme health centres were trained in the principles and practice of syphilis control in pregnancy.

Partner notification and treatment: To facilitate partner referral a standard form for partner notification was developed wherein the partner was invited to come to the clinic for reasons related to the pregnancy. Each sero-reactive woman was asked to bring her partner(s) to the clinic for treatment. Partners of sero-reactive women were treated presumptively.

Supervision and monitoring: A team of trained supervisors from the NCC and its partners used a standardized form. The forms required the supervisors to state the objective of the visit, the topics discussed and findings from evaluation of the laboratory, stock management, clinic staff on duty, record keeping, quality control and partner tracing activities.

(Source: Jenniskins et al, 1995) 


\section{CASE STUDY METHODOLOGY}

This case study of the decentralised model was undertaken for two reasons. First, during the pilot project itself, the NCC was financially responsible for supporting the health facilities and personnel, and for ensuring that drugs for treating positive cases were available, while the other partners financed the supply of equipment, reagents, training and technical assistance. Since the pilot project, most NCC clinics have benefited from a World Bank and DfID-funded project that supports STI training and drug supply through the national STI prevention programme.

Over time, financial and technical support from these partners has been gradually withdrawn and the NCC left to finance and manage the activities within its regular programme and budget. This has raised concerns about the sustainability of the decentralised programme, and so the NCC supported the case study in order to obtain information to guide its future planning for antenatal syphilis screening and treatment services.

Second, the initial success of this decentralised approach makes it very relevant to those countries in Africa and elsewhere that see antenatal syphilis detection and treatment as a priority in the battle to contain the AIDS epidemic. This case study was undertaken to document how the model has been implemented, and its effectiveness eight years after it was first introduced. The objectives of the case study were to:

\section{Syphilis screening and management at standard NCC clinics (not implementing the decentralised model)}

Screening: Women receiving ANC at the NCC clinics not participating in the pilot program are referred to the nearest pilot program clinic for syphilis testing. In some cases, blood is taken and sent to the nearest pilot clinic for testing. If it is the woman who is referred, she is also responsible for returning the results to the referring clinic.

Treatment of positive clients: If the client is referred to the pilot clinic for testing, she has the option of getting treated at the pilot clinic in which case she is given a note to take back to the referring clinic indicating that she has been treated. Otherwise she is treated at the referring clinic when she returns with the result. In cases where the specimen is sent by the referring clinic, the client gets treated at the referring clinic once the results have been received from the testing sites.

Partner notification and treatment: The clinics are expected to follow the same procedures as those used at the pilot clinics where clients are counselled and issued a partner notification slip for inviting their partners to come for treatment.
- Ascertain the readiness of NCC antenatal care services to continue providing syphilis screening and treatment.

- Evaluate the quality of antenatal care services in general, and syphilis screening and management specifically.

- Assess the performance of the syphilis screening and treatment programme.

- Identify lessons learned from the implementation of the decentralized syphilis screening and treatment programme.

To achieve these objectives, the case study was designed to document antenatal syphilis screening activities in the decentralised clinics, and to compare them with the standard antenatal syphilis screening procedures in a sample of those clinics that have adapted the decentralised 
model. The box on the previous page describes the antenatal syphilis screening procedures followed in the standard clinics.

Of the 14 clinics that have adapted the decentralised approach, three were closed for renovations at the time of the case study, and so all eleven functioning clinics were included. Seven clinics serving a similar population within the NCC system were selected from the remaining $40 \mathrm{NCC}$ clinics that had not participated in the pilot programme (see Appendix 1 for a listing of the 18 clinics included in the study).

Data were collected in March - April 2000 using two teams of trained interviewers, each comprising six persons: four experienced nurse-midwives and two research assistants, using the following methods.

Observations of Client/Provider interaction: All consultations with women attending antenatal clinics for the first time, and with every third revisit client, were observed. A trained observer recorded all interactions between the client and the provider using a pretested checklist. In total, 611 consultations, 329 first visits and 282 revisits were observed.

Exit Interviews with ANC clients: All clients whose consultations had been observed and who gave their informed consent were interviewed as they left the clinic. They were asked about their experiences and perception of the antenatal and syphilis screening services that they had just received by a trained interviewer using a pretested questionnaire.

Patient Flow Analysis: Each client who attended the clinics for antenatal care services was issued with a Patient Flow Analysis (PFA) form as they entered the clinic compound. The client carried this form to all the clinic rooms in which she was attended to and each provider recorded the time that the clients handed the form to them and then the time that they finished attending to the client. The form was collected from the client as she left the clinic by an interviewer who recorded her time of departure from the clinic. A total of 1,298 clients were included in the PFA.

Focus Group Discussions: Fifteen focus group discussions (FGDs) were conducted with service providers to gather information on their experiences and perceptions with providing antenatal care services generally, and syphilis screening and management specifically. The FGDs were conducted for staff at all clinics except three.

In-depth Interviews: In-depth interviews using structured formats were conducted for key actors involved in the pilot study, and/or who are responsible for the current antenatal syphilis-screening programme. The interviews were used to obtain information on the programme's development, implementation and achievements as well as its sustainability and future trends. The key informants included: the NCC Medical Officer of Health, two Assistant Medical Officers of Health (AMOH) at City Hall, two at the divisions and one at the Special Treatment Centre (STC). One sister-in-charge of supervision at the division and two the STC were also interviewed.

Abstraction of service statistics: Clinic records including daily registers, STI management registers and client record cards were reviewed and information abstracted using a structured form to compile service statistics for a period of twelve months preceding the study. These data were analysed to determine the number of women attending for antenatal care, types of visits, number tested and/or referred for syphilis testing, number of women testing positive for syphilis infection who were treated and number of partners of positive women treated. This 
information provided a crude estimate of the burden of syphilis infection among antenatal clients and some insight on how the NCC was managing maternal syphilis infection.

Cost analysis: Information on the supplies and their costs used to collect blood, as well as the laboratory costs to test the blood for syphilis was obtained through interviews with programme managers and service providers and quotations from suppliers. In addition, information on other supplies used for antenatal visits that were not specifically related to drawing blood (such as costs for collecting urine and giving tetanus injections) was also collected using the same sources. Data on the salaries and benefits of the personnel who either provided services to the women making the antenatal visits or supervised the staff who provided these services at the six pilot clinics was obtained from the personnel department at the NCC. The time spent providing services to clients was estimated using information from the client flow analysis. This information was used to calculate the costs associated with direct labour, indirect labour, capital and clinic infrastructure, support staff time and clinic supplies.

The results from the case study are presented as follows:

- Readiness of NCC health facilities to provide general antenatal services and syphilis screening and management services.

- Implementation of syphilis screening and management services.

- Performance of the decentralized syphilis screening and management programme. 


\section{READINESS TO PROVIDE ANTENATAL CARE AND SYPHILIS SCREENING AND TREATMENT}

\section{Service Provider Guidelines On Antenatal Care}

The MOH revised its Reproductive Health/Family Planning Policy Guidelines and Standards for Service Providers in 1997 and launched them in early 1998; these guidelines include instructions on how antenatal care should be provided. Only one of the 18 study clinics had a copy of these guidelines, and most providers were not aware of their existence.

During the FGDs, service providers said, "The client antenatal clinic card guides us on the clinical procedures and the information to ask the client". At most clinics, however, client record cards were not available and service providers were using improvised notebooks to record client information. In such circumstances, the providers said that they rely on knowledge acquired during basic medical training and their experience on the job to assess and provide services to the clients.

\section{Statements made by service providers}

"We do not have service provider guidelines but as far as we are trained we know what we should do according to our training."

"The way we were taught in midwifery, that is how we provide. We know exactly what we are supposed to do."

"If you have stayed in the council for long...this is like a routine."

"With the knowledge that one has, that is how we go about attending to these mothers...you know exactly what you are supposed to do."

"It depends on the clinic where you are working ... you will see the necessary things that you should do for antenatal client".

According to the Medical Officer of Health in charge and other key informants, the NCC Public Health department does not have specific guidelines for the provision of antenatal care and/or for syphilis screening and treatment. These key informants also said that service providers are trained in midwifery and provide these services daily, implying that they should know what is expected of them.

These findings are not surprising given that none of the key informants had seen the revised Guidelines and Standards. In addition, coverage of antenatal care in this 68-page document is minimal. Antenatal care is presented in one and a half pages compared to 57 pages devoted to family planning services. This suggests that antenatal care generally has not been given the attention it deserves within a comprehensive reproductive health services package, and the lack of clear guidelines is likely to affect the quality of services provided to antenatal clients. 


\section{Service Provider Guidelines For Syphilis Screening}

Similarly, there are no service provider guidelines on syphilis screening and testing for women attending antenatal care. This was the case even at the pilot project clinics, where no written guidelines were available for the service providers. Service providers and key informants indicated that because syphilis is an STI, syphilis screening is considered part of STI management and not antenatal care. They all mentioned that guidelines for syphilis management are covered in the syndromic case management flow charts provided by the $\mathrm{MOH}$. According to one provider: "We have the flow chart in the STI room whereby you can use the syndromic approach." In support of this, the MOH said "there are standardised guidelines from the Ministry of Health on syndromic management of STIs which explain screening and treatment procedures for both ANC clients and their partners".

However, the syndromic management flowcharts have instructions for the management of genital ulcer disease syndrome, and not syphilis specifically. Consequently, there are no specific instructions on detecting and managing syphilis infection among antenatal clients, and/or asymptomatic women who may have syphilis infection in the secondary and/or tertiary stages. The flowcharts also do not provide any guidance on how to manage infants of mothers with genital ulcer disease, or who test positive for syphilis infection during pregnancy. In addition, the revised reproductive health service provider guidelines do not include syphilis screening, diagnosis and treatment as part of the services to be provided to antenatal clients.

\section{Equipment And Supplies For Providing Antenatal Care}

According to the $\mathrm{MOH}$ service delivery guidelines, all health facilities should have, at a minimum, the equipment described in Table 1. As the table shows, however, less than one half of the pilot clinics had a fetoscope, functioning BP machine, and examination lights.

\section{Table 1 Percentage of clinics with essential equipment for antenatal care}

\begin{tabular}{|l|c|c|}
\hline Equipment & $\begin{array}{c}\text { Pilot clinics } \\
(\mathbf{n}=\mathbf{1 1})\end{array}$ & $\begin{array}{c}\text { Standard clinics } \\
(\mathbf{n}=\mathbf{7})\end{array}$ \\
\hline Adult weighing scale & 100 & 71 \\
\hline Disposable gloves & 81 & 57 \\
\hline Stethoscope & 81 & 71 \\
\hline Sterilizer & 54 & 42 \\
\hline Sterile specula & 36 & 57 \\
\hline Fetoscope & 27 & 57 \\
\hline BP machine & 27 & 42 \\
\hline Examination light & & \\
\hline
\end{tabular}


Pregnant women in Kenya are supposed to be routinely given ferrous sulphate and folic acid tablets to supplement their nutrition, anti-malarial drugs for malaria prophylaxis, and antitetanus toxoid vaccine to protect the woman and foetus from tetanus infection. Over 90 percent of all clinics had supplies of anti-tetanus toxoid vaccine, but less than one quarter had anti-malarial drugs. Most pilot programme clinics were stocked with supplies of ferrous sulphate and folic acid, whereas less than one-half of the standard clinics had these nutrition supplements.

Provision of these medications was not part of the pilot study, and so it is not clear why there is this difference between clinics. Explanations offered by service providers and policy makers are that staff at the pilot clinics were trained in logistics management and thus were more likely to order and stock all medications required for antenatal care, and the pilot clinics were also supervised more frequently. The lack of anti-malarial drugs is thought to be due to the fact that Nairobi is not considered an endemic area for malaria and therefore most clinics would not routinely stock these drugs.

\section{Equipment And Supplies For Syphilis Testing And Management}

Pilot clinics were initially provided with the basic equipment and supplies required for carrying out the RPR test, and Table 2 shows that most pilot clinics still have the centrifuge, RPR card shaker/mixer, and a regular supply of RPR test cards. One clinic not included in the pilot study also had a centrifuge and shaker, which was acquired through participation in another research study with the University of Nairobi.

Functioning equipment and supplies needed to draw and store blood for testing were available at most, but not all, pilot clinics. Although, needles and syringes, swabs and disinfectant were missing at a significant number of both pilot and standard clinics, the majority (72\%) had vacuutainers that could be used to draw and transport blood specimens for testing. The shortage of both syringes/needles and vacuutainers at the standard clinics implies that blood cannot be taken for testing and that medication through injection cannot be provided, thereby severely limiting the readiness of the standard clinics to undertake these two components of the decentralized approach.

Consequently, clients cannot be screened or treated, or they have to purchase these items themselves. Indeed, several clients were observed being sent by providers to purchase needles and syringes. Some service providers said that they disliked asking clients to purchase these supplies; "We feel that we are not doing the right thing by telling them to go and buy a syringe and a needle. You know, even if it is cost sharing, we are supposed to have these things."

The lack of swabs and surgical spirit also means that although clients may bring syringes and needles, providers may be collecting blood or treating syphilis without practising infection prevention procedures. Commenting on this, providers in one clinic said, "Like now we are doing immunization and we have no spirit, yet we know that we are supposed to use spirit but, what do we do? We are using water. And we can see the mothers keep asking themselves, why don't you have spirit? Telling them to go and buy all the things makes us feel we are overloading them." But other providers said, "If one is in the injection room, and there is no cotton wool and spirit, we ask the patients to contribute two shillings (0.03 US\$) each and buy the things for themselves and then I inject them." 


\section{Table 2 Percentage of clinics with essential equipment and supplies}

for syphilis screening

\begin{tabular}{|l|c|c|}
\hline Equipment and supplies & $\begin{array}{c}\text { Pilot clinics } \\
(\mathbf{n}=\mathbf{1 1})\end{array}$ & $\begin{array}{c}\text { Standard clinics } \\
(\mathbf{n}=\mathbf{7})\end{array}$ \\
\hline RPR card shaker & 100 & 14 \\
\hline Centrifuge & 91 & 14 \\
\hline RPR cards & 81 & 57 \\
\hline Cooler box for specimens & 72 & 0 \\
\hline $\begin{array}{l}\text { Vacuutainers/specimen } \\
\text { bottles }\end{array}$ & 55 & 42 \\
\hline Sharp disposal container & 55 & 29 \\
\hline Surgical spirit/disinfectant & 45 & 57 \\
\hline Refrigerator & 45 & 42 \\
\hline Swabs & 09 & 14 \\
\hline Emergency resuscitation \\
tray
\end{tabular}

Service providers have had no choice but to ask clients to supplement the irregular supplies received from the $\mathrm{MOH}$ stores. The NCC policy makers and programme managers are aware that clinic staff ask clients to contribute money to purchase supplies or to come with them, but have decided to let it continue as long as the money collected is used for the right purpose. Based on the willingness and ability of clients to make these contributions, the NCC managers are considering the possibility of formalising these payments in the form of a costsharing strategy.

The MOH currently recommends that all clients testing positive on an RPR test be treated with a single dose of benzathine penicillin. For clients who may be allergic to penicillin, erythromycin should be used. For treating clients who may have evidence of secondary and/ or tertiary syphilis, procaine penicillin is recommended. As can be seen in Figure 1, the pilot clinics were more likely to have such supplies, but were also more likely to have procaine penicillin than benzanthine penicillin.

Although the standard clinics do not provide on-site screening for maternal syphilis, they are expected to provide on-site treatment for clients they refer for tests and who come back with positive results. This has been possible since most of the service providers in the NCC clinics have had some training in the management of STIs using the syndromic approach, which 
includes management of genital ulcers. However, as can be seen from Figure 1, less than one half of the standard clinics are in a position to do this. Given that the NCC supplies both pilot and standard clinics with drugs from a central source, the differences in drug availability may be due to the logistics training and supervision provided to the pilot clinics rather than to a problem with the central procurement system.

Figure 1: Percentage of clinics with drugs for treating syphilis

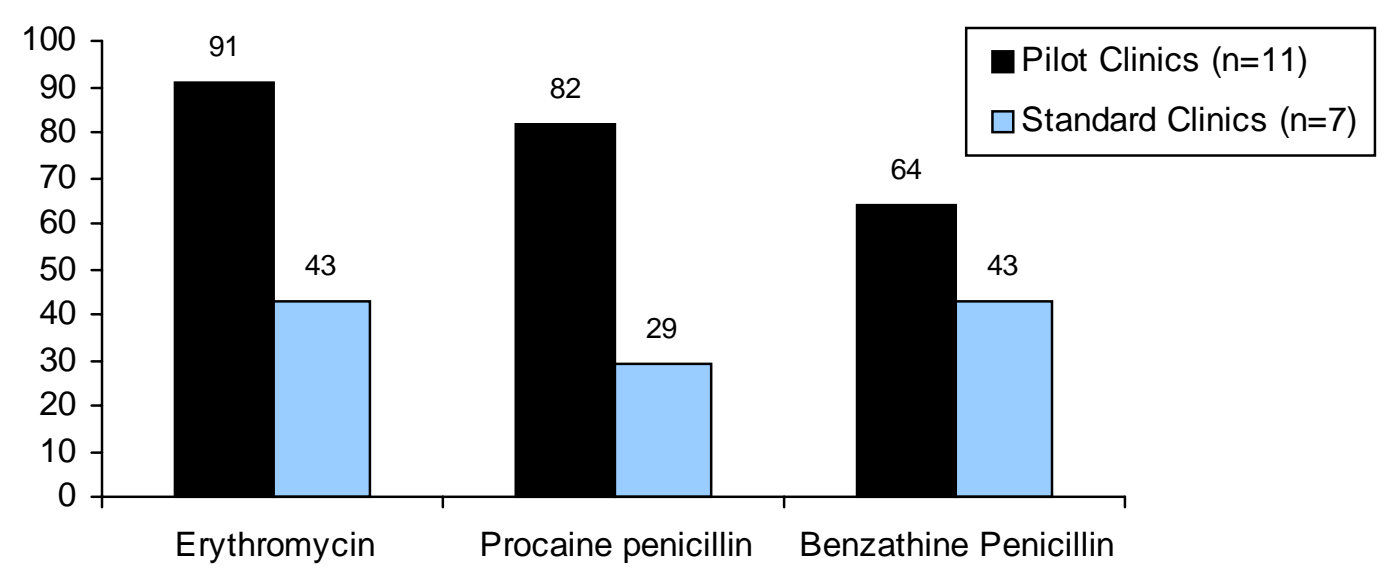

\section{Information, Education And Communication Activities}

Providers are expected to give group health talks every morning before commencing services, but such talks were observed at only four clinics. The topics covered during these four health talks included: the importance of early antenatal care and syphilis testing in pregnancy; complications of untreated syphilis to the unborn child; transmission of HIV/AIDS from the mother to the unborn child; and voluntary counselling and testing. Asked why health talks are rarely given, the providers said:

"Sometimes when we report on duty, the clients are so many that our priority is just to clear the queues."

The vast majority $(94 \%)$ of clinics had at least one type of poster and more than one half of these had messages on HIV and STI transmission and

\section{Table 3: Availability of IEC Materials}

\begin{tabular}{|l|c|c|}
\hline Posters with themes on: & $\begin{array}{c}\text { Pilot clinics } \\
(\mathbf{n}=\mathbf{1 1})\end{array}$ & $\begin{array}{c}\text { Standard clinics } \\
(\mathbf{n}=\mathbf{7})\end{array}$ \\
\hline Prevention of STIs & 82 & 29 \\
\hline Prevention of HIV/AIDS & 73 & 57 \\
\hline Transmission of STls & 64 & 57 \\
\hline Transmission of HIV & 55 & 57 \\
\hline $\begin{array}{l}\text { Other themes } \\
\text { Complications of untreated } \\
\text { STls }\end{array}$ & 46 & 0 \\
\hline
\end{tabular}
prevention. Fewer than 
10 percent of the pilot clinics had any posters with messages on how STIs can be treated, and none had any posters with specific messages on antenatal care or maternal syphilis. Posters with messages on child nutrition, ophthalmia neonatorum and polio were found in nearly one half of both types of facilities.

Programme managers and service providers cited two possible reasons for the absence of posters with themes on antenatal care: the lack of appropriate posters, and a lack of funds for producing them because production of IEC materials is normally carried out using donor funds.

\section{Staff Training And Experience}

During the pilot project, 69 nurses in the nine clinics were trained in screening of antenatal women and management of maternal syphilis. Of these 69 nurses, only 12 are still serving at the project sites, the remainder having either left the $\mathrm{NCC}$ altogether or been transferred to other health facilities within the NCC.

The nurses trained during the pilot project were expected to provide on-the-job training for their colleagues following a one-day induction course conducted by the NCC. Thirty nurses now working at the pilot clinics had been trained through this approach. Thus, of the 114 nurses providing antenatal care services at the time of the case study in both pilot and standard clinics, only 42 had received any form of training in maternal syphilis screening and management, and nurses trained by fellow nurses formed the majority of these. All but one of the 42 trained nurses were based at the pilot clinics, and so most nurses providing antenatal care services at the NCC clinics have not received adequate training in maternal syphilis screening and management. One of the key trainers said "We trained the nurses in RPR for half a day to one day. We used to go round to the sites with laboratory technicians. When the EEC took over the programme, we recalled the staff here and trained them for one day because those trained had been transferred or retired".

All providers felt that the training received was adequate to perform their duties. However, providers who received training at the STC indicated that they did not cover pre- and post test counselling. A few providers also said that the training did not cover "confirmatory test" techniques. The providers felt that they need regular updates on syphilis screening and that it should also include training on HIV/AIDS testing also.

Nevertheless, service providers appeared to have good knowledge of the procedures involved in syphilis testing and management. In the FGDs providers reported that they are expected to do the following:

- Draw blood from the client

- Put blood in the centrifuge and spin it in a rotator for about 5-9 minutes

- Leave blood to settle in order to separate it from the serum

- Draw blood with a pipette and drop it on RPR cards which bear the client's identification numbers

- Drop the reagent on each circle in the card

- Read the results. 


\section{IMPLEMENTATION OF THE DECENTRALISED MODEL}

\section{General Antenatal Care}

Observations of 312 new antenatal clients (249 at the pilot clinics and 63 at the standard clinics) revealed that they received variable levels of clinical assessment during their consultation (see Figure 2). Interestingly, new antenatal clients attending the standard clinics were more likely to be examined for various conditions than those attending the pilot clinics except for weight. Although one would have expected the opposite situation, it is important to note that the syphilis screening and management training did not include general antenatal care.

\section{Figure 2 Percentage of new antenatal clients assessed by condition}

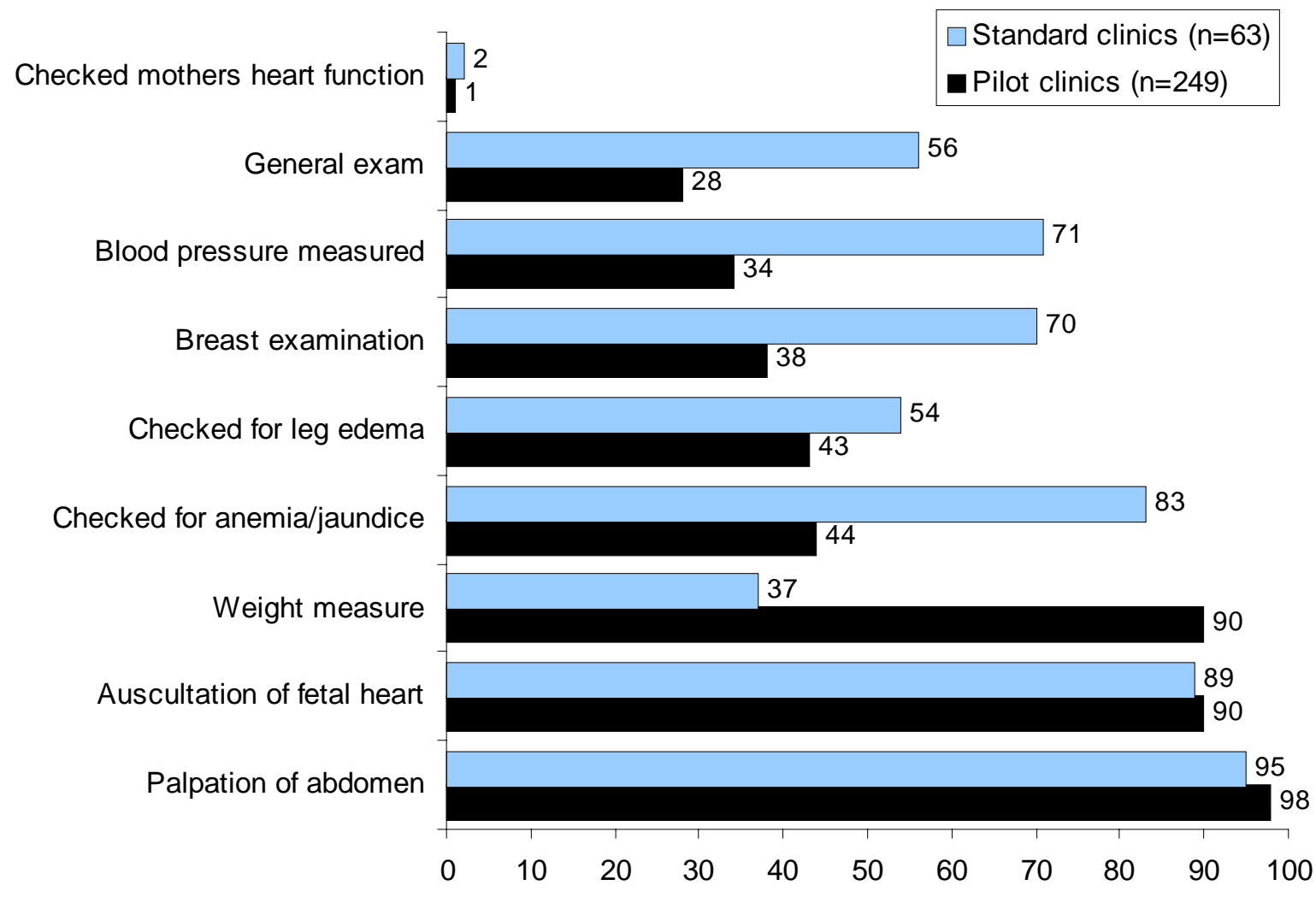

Service providers stated that they do not always undertake some of these procedures because they do not have the necessary supplies and equipment and not because they do not know what procedures are expected. However, they also indicated that current $\mathrm{MOH}$ regulations do not allow nurses to carry out certain examinations, like checking for maternal heart function.

Some clients are also aware that they are not being adequately assessed during the clinic visits. One client commented: "You know, I am due in the first week of April but I have never been weighed and my blood pressure has never been taken though I see it being done in other clinics." While another client commenting to her friend said: "I had come to be taken blood pressure because I am not feeling well but I hear there is no blood pressure machine."

Providers reported that they use the client card to guide them when taking history and examining clients, but as can be seen in Figure 2, they do not strictly follow the procedures 
stipulated on the card. When asked why not, one provider replied, "If you follow the card, you will spend the whole day here". ${ }^{1}$ Not all clinics were issuing cards to their clients due to stock-outs, and clients were being asked to buy their own notebooks instead.

As Figure 3 shows, less than one-half of the new antenatal clients attending the pilot clinics received information that is considered essential for any pregnant woman. The situation was similar at the standard clinics, although a larger proportion was likely to be counselled than at the pilot clinics.

These findings may explain why clients' knowledge of pregnancy-related dangers was poor. During exit interviews, the proportions of clients knowing the key signs of problem pregnancies were low, i.e. pain in the lower abdomen (50\%), bleeding (36\%), vomiting (28\%), lack of foetal movements (18\%), and dizziness (11\%). Moreover, fewer than one half of the repeat clients observed were counselled about these topics, and they were even less likely to be counselled compared to the new clients. Some providers said that they do not fully counsel clients because of large client loads and poor motivation.

\section{Figure 3 Percentage of new antenatal clients counselled about pregnancy}

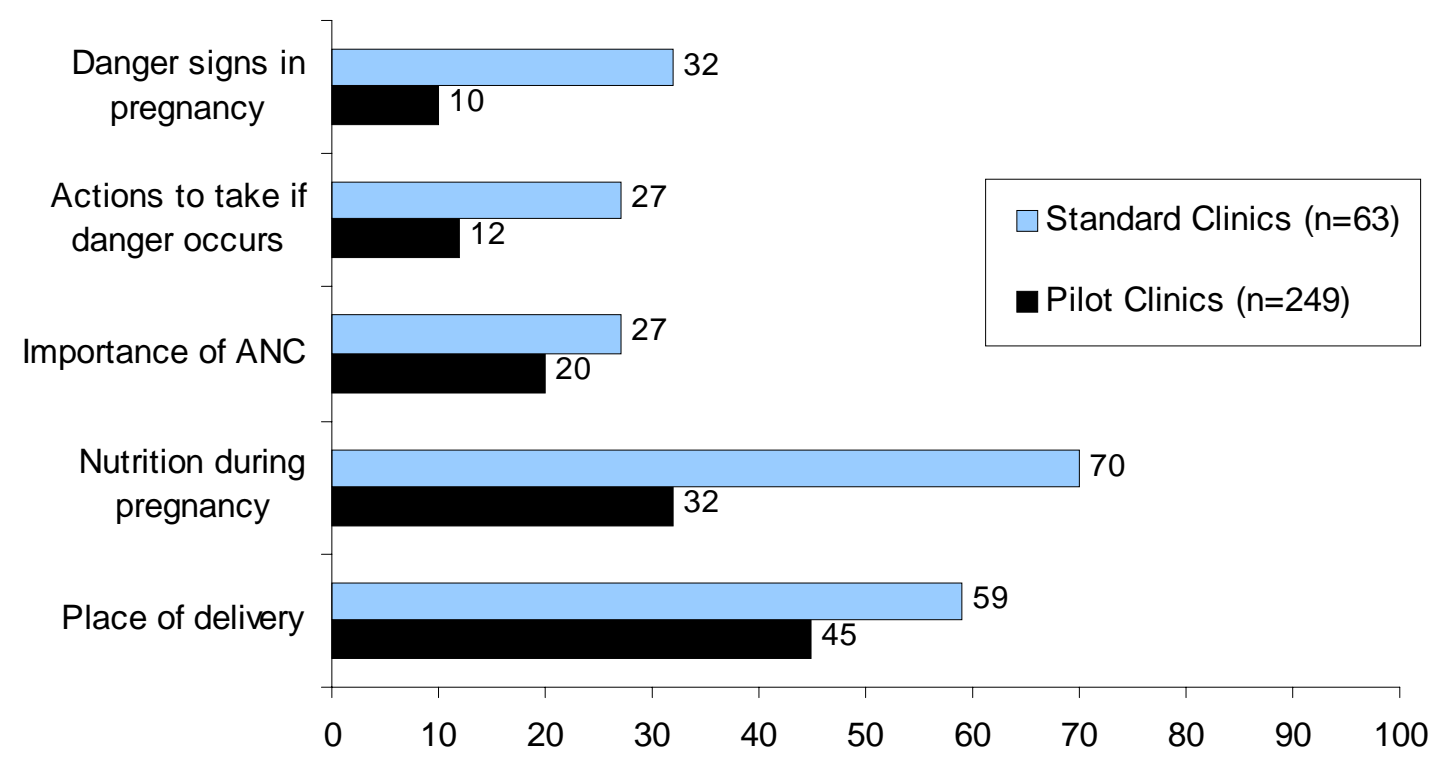

\section{Syphilis Screening}

Although there are no $\mathrm{MOH}$ or NCC guidelines for syphilis screening, the pilot project recommended that the first RPR test should be done at 20 weeks or earlier and repeated again at 28 weeks. However, this is difficult to implement because women do not turn up at the clinic early enough; on average, clients made their first antenatal clinic visit at 26 weeks, with a range of 18-28 weeks. Providers also said that carrying out two tests for each woman was not possible because of the frequent shortages and irregular supplies of RPR test kits and other supplies required to draw the blood for testing.

\footnotetext{
${ }^{1}$ The introduction of standard checklists as job aids to help providers during family planning and antenatal consultations has been recommended as a way of improving job performance (Solo et al, 1998, Maggwa et al, 1999), but for antenatal care it appears that checklists alone may not make a difference if there has not been adequate training and support.
} 
Providers also indicated that women who are not tested during their first visit are likely to go through the pregnancy without being tested at all. According to one provider: "Once it is not done in the first visit, it may not be done in the subsequent visits because when these clients come for subsequent visits, they will be treated as revisits and priority for testing is given to first visits.”

Although blood testing for syphilis was universally done during the initial phase of the pilot project, it is no longer undertaken all the time. During the case study, only 40 percent of the first-visit antenatal clients at the pilot clinics, and 16 percent of those attending the standard clinics were observed to have blood taken for testing (Figure 4). Only four percent of the revisit clients were tested for syphilis. Clients not tested during the initial visit at the pilot clinics were either given an appointment to return when the clinic would be able to carry out the test, or were referred to another pilot clinic where they could get the test done.

\section{Figure 4 Percentage of new ANC clients assessed for syphilis by type of assessment}

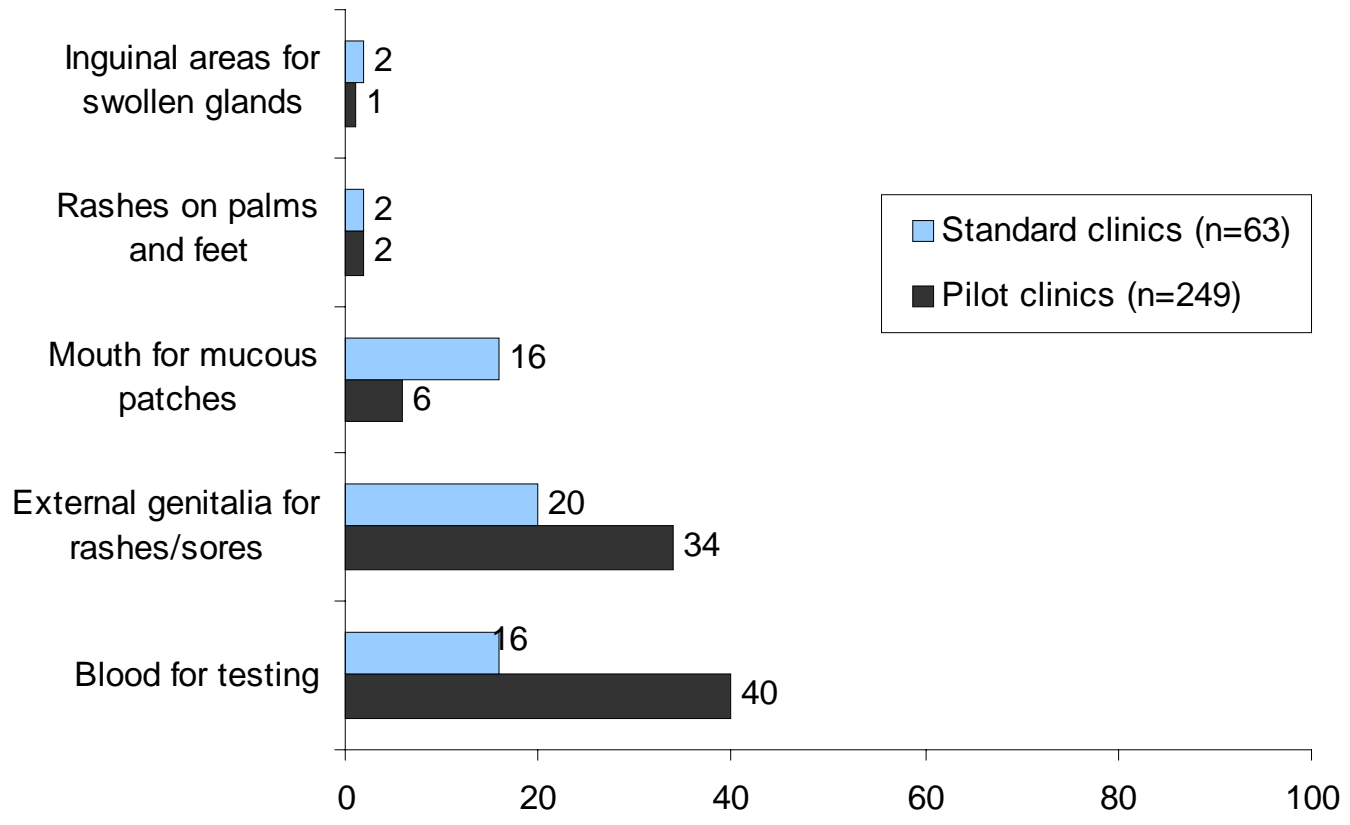

A major reason for the pilot clinics not carrying out syphilis testing was the lack of needles and syringes with which to draw blood. Moreover, at the time of the case study Nairobi was experiencing severe power rationing that affected many of the clinics and so the rotator/mixer and centrifuges required for RPR testing could not always be used.

According to providers, examination of the genitalia is no longer routinely done for two reasons: a shortage of supplies for pelvic exams (e.g. gloves, specula); and the introduction of syndromic management flow charts. "In the past, we used to even check the vulva but these days we usually ask the mother whether she has a problem like itching or discharge and if she tells you she has discharge, you check by looking what type of discharge she has, whether it is yellow or normal discharge and then treat."

After history taking and clinical examination, each new antenatal client is referred to a procedure room where a nurse trained in drawing blood and carrying out the syphilis test draws her blood for the test. The client is then asked to wait outside while the specimens are 
being processed. To maximize use of the RPR card, which can be used to test up to 10 specimens at once, specimens from several clients are tested in batches. This means that often clients have to wait while other clients have their blood drawn before they can get their results and treatment.

At clinics with laboratory technologists, they are responsible for drawing blood and performing the tests. In the standard clinics where the tests are not available on-site, clients are referred to the nearest pilot clinic that provides blood testing. In some instances, the clients are referred only for the test and are required to bring the results to the referring clinic for treatment, while in other cases they are referred for both the test and treatment. The decision on what type of referral to undertake is mostly influenced by the availability of drugs at the referring clinic.

\section{Counselling Clients On Antenatal Syphilis}

For a successful antenatal syphilis prevention and control programme, clients also need to have a good understanding of the disease, its transmission and its implications. According to the service providers, "We counsel before you (we) remove blood, tell them about the signs and symptoms, complications, treatment and the importance of treating the client and the contact". They added that they inform clients about complications resulting from untreated syphilis such as abortion or deformity in the baby.

However, during observations of client-provider interactions it was found that 62 percent of new antenatal clients attending the pilot clinics were not counselled at all about maternal syphilis and an even larger proportion (74 percent) were not counselled at the standard clinics. Although clients who had their blood taken for testing were more likely to be counselled (55 percent) than those who did not have their blood taken ( 25 percent), it is important to note that almost half of the women whose blood was taken for testing were not counselled on any aspect of maternal syphilis.

\section{Figure 5 Percentage of new antenatal clients informed about syphilis}

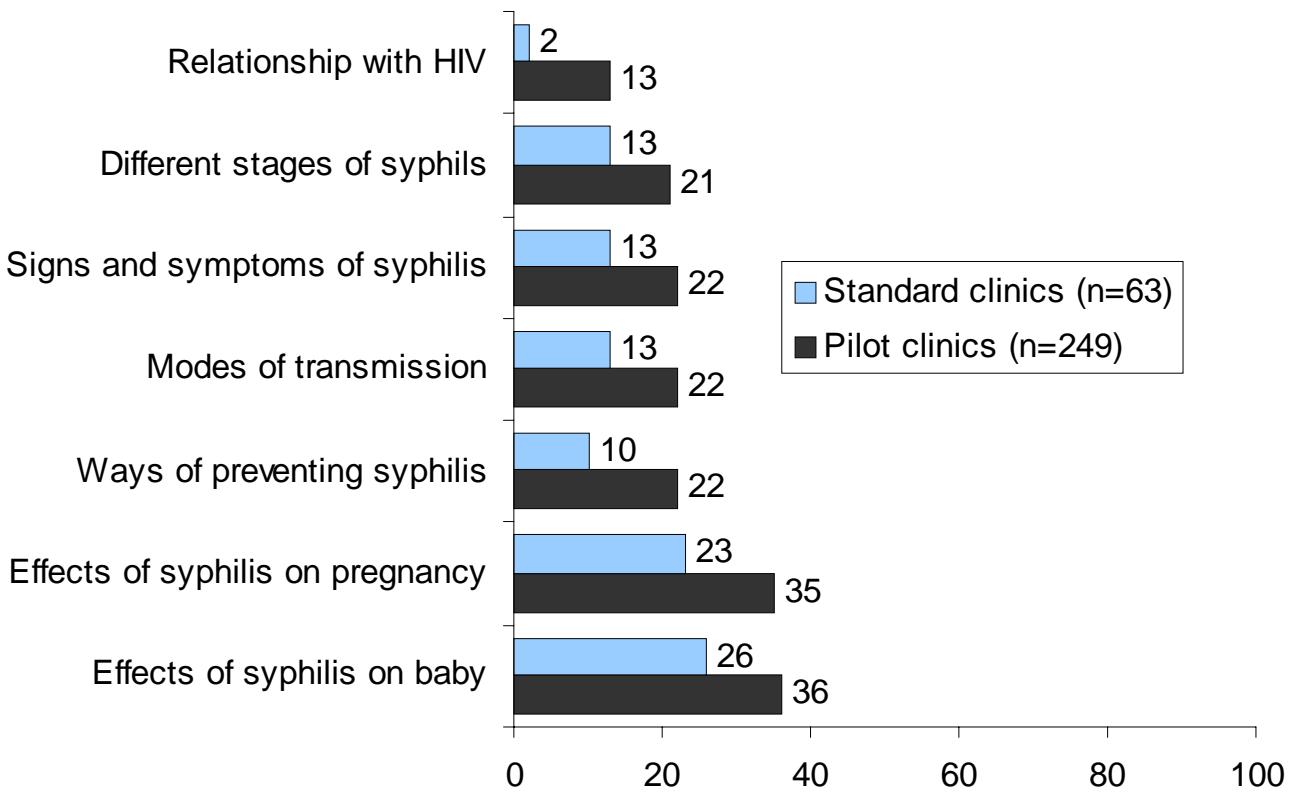


For the clients who were counselled about maternal syphilis the most commonly discussed topics were the effects of the infection on the baby and on the pregnancy (see Figure 5). The direct relationship between syphilis infection, genital ulcers and HIV infection was discussed with only a small proportion of the clients.

Clients' knowledge about syphilis, however, was reasonable given that a large proportion were not counselled during the consultations. Most of the clients (82\%) knew that syphilis is transmitted through unprotected sex, and more than two-thirds (71\%) were aware that syphilis could be transmitted from the mother to the unborn child during pregnancy. Smaller proportions knew that this could lead to abortion (43\%), a deformed baby (41\%) or stillbirth $(10 \%)$. Nearly two-thirds said that avoiding multiple sexual partners can prevent syphilis, and the use of condoms was mentioned by 40 percent.

Approximately the same proportions of clients were counselled about STIs generally as well as HIV/AIDS, including signs and symptoms, transmission and prevention. Among all clients, 18 percent were informed about Voluntary Counselling and Testing (VCT) for HIV/ AIDS, but only 14 percent were told about using condoms for STI prevention.

\section{Infection Prevention Procedures}

During the case study, 122 new and revisit clients were observed to have blood taken (see Figure 6). Of the 122 cases observed, 110 were at the pilot clinics and 12 at the standard clinics. For 40 percent of these clients, service providers did not wear gloves when drawing blood, and providers at the standard clinics were less likely to wear gloves than those at the pilot clinics (8\% and $66 \%$ respectively).

The other major difference was in the disposal of used needles and syringes. Service providers at the standard clinics were less likely to use a sharps disposal facility for needles and syringes than those at the pilot clinics (8\% and $27 \%$ respectively). Moreover, although gloves were worn when drawing blood from 60 percent of the clients at the pilot clinics, the gloves were not regularly changed between clients. Given the high prevalence of HIV infection in the country, failure to adhere strictly to standard infection prevention procedures is worrying.

\section{Figure 6 Percentage of cases in which infection prevention procedures were followed during blood drawing}

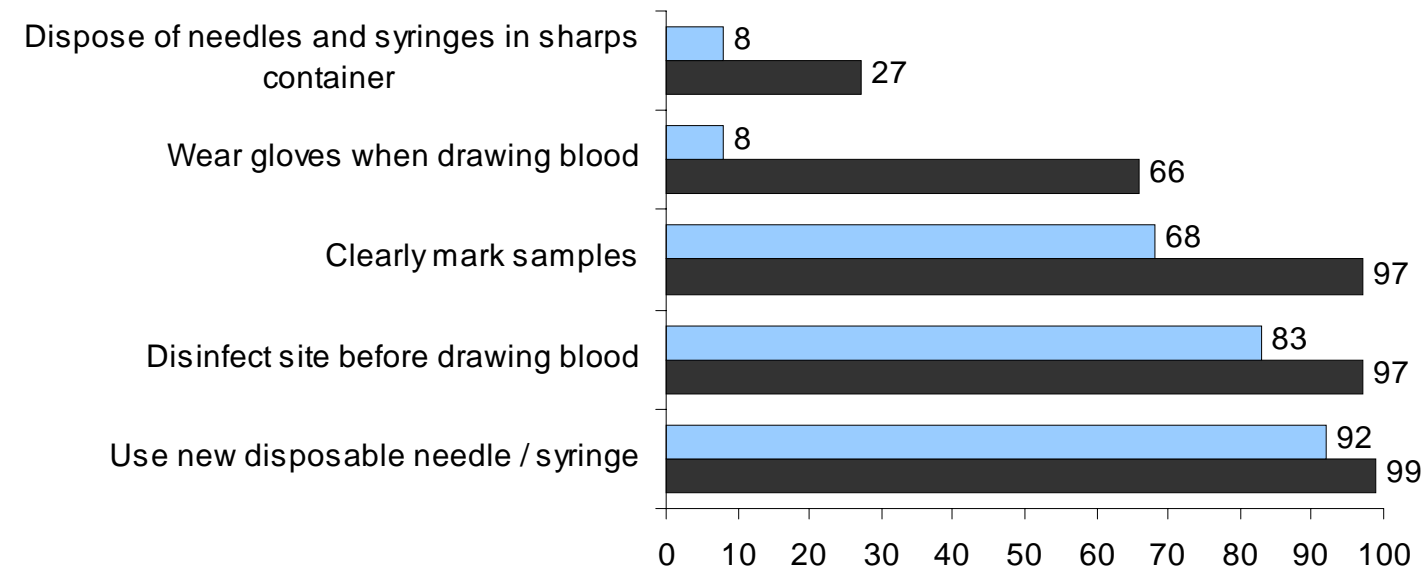

Pilot clinics $(\mathrm{n}=110) \square$ Standard clinics $(\mathrm{n}=12)$ 


\section{Treating Positive Women}

Providers said that the test results are usually available to the clients immediately if the test is carried out at the clinic (or within twenty-four hours if there is power rationing). After the blood has been tested in the procedure room, clients are called back into the room and informed of their results.

Those who test positive are treated using a single dose of Benzathine Penicillin, or Erythromycin if allergy to penicillin is suspected. Benthazine Penicillin 2.4 MU is given by deep intra-muscular injection as a single dose treatment. Although allergy to penicillin is rare, clients with allergy are treated using Erythromycin 500mg taken three times a day (TDS) for two weeks. Penicillin allergy is identified through asking positive women whether they have ever reacted to an injection or any drug in the past.

Positive clients are then counselled about partner notification and issued a partner notification slip. The nurse records all information about the client, test results, treatment and partner notification in an RPR register kept in the same room. In some clinics, this information is kept in a common register for antenatal clients.

Unfortunately, during the case study it was not possible to observe how the results of the syphilis test were conveyed to clients or how positive women were managed because another study (on HIV in pregnant women) was temporarily disrupting standard procedures.

\section{Notification And Treatment of Partners}

Service providers reported that the standard partner notification slip developed during the original pilot project is still being used in most clinics. For all clients testing positive, the nurse completes the partner notification slip (See box) and gives it to the client, after counselling her on the importance of partner notification and treatment. The client is also given some guidance on how to inform the partner about the need to visit the clinic. According to the providers interviewed, mothers are advised not to reveal to the partner/ husband the reason for their being asked to come to the clinic because if they are told the truth, they may refuse or be afraid to come. As one provider indicated: “...I tell the client to tell him to come because I have an issue I want to share with him." "Because of their concern for the baby, they always come," observed one of the key informants interviewed.

The providers indicated that when the partner comes to the clinic, he is seen in private and explained the reason for his visit. The providers added that it is not mandatory for the partner to come with the client, but if

\section{NAIROBI CITY COUNCIL}

$$
\text { CONTACT SLIP }
$$

Date

Name of Clinic

Index Clinic No.

Index Diagnostic Code

Please come to this clinic as soon as possible for medical advice

Tafadhali njoo kliniki hii haraka iwezekanavyo

Signature of Clinician

(Similar services available in the clinics listed overleaf)

(Huduma kama hizi hupatikana pia katika kliniki zifuatazo) 
they do come together they are counselled together. After counselling, the providers give the partners the same treatment that is given to the client. In situations where the woman's partner has more than one partner, providers advise him to bring the other partners for treatment. At clinics where there are no contact slips, the providers reported that they send a verbal message to the partner/husband, but this has proved ineffective because they hardly ever get to know whether the partner came for treatment.

As can be seen from the sample notification slip, the partner does not have to come to the same clinic as the index client; they can visit any of the clinics listed on the back of the slip. However, when this happens, the clinic attending to the partner is expected to send the notification slip back to the index clinic so that by using the Index Clinic number on the slip the outcome can be linked to the index client.

\section{Assessing Clients' For Other Reproductive Tract Problems}

Observations of client-provider interactions showed that service providers rarely carry out any risk assessment for STIs generally, including for HIV. Only nine percent of new antenatal clients were asked for information about their behaviour or lifestyle that would have been useful in determining their risk status. Although data from other studies in Kenya and the region indicate that risk assessment only marginally improves the effectiveness of syndromic management (Solo et al, 1998; Maggwa et al, 1999; Welsh et al, 1997), failure to ask a client about her and her partner's sexual relations and history of STIs represents a missed opportunity for providing education and counselling on STI and HIV/AIDS prevention.

As can be seen on Figure 7, approximately one-third of new antenatal clients were asked about abnormal vaginal discharge and had their external genitalia examined. More surprising was the observation that even when some clients complained about a discharge or itching, symptoms suggestive of a reproductive tract infection, the providers did not ask for more information or carry out any examinations to confirm or discount the complaints. For example, of the 14 percent $(n=181)$ of clients who reported symptoms suggestive of an RTI (genital discharge, bleeding, itching, ulcers or lower abdominal pain) only one-third of them subsequently had a pelvic examination, and the syndromic approach was not used to manage their symptoms.

Cancers of the reproductive tract are common among women in the reproductive age group in Kenya and antenatal clinics provide an opportunity for screening women to increase the chances of early detection. The Pap smear or a visual inspection of the cervix during a speculum examination can be used in identifying abnormal lesions of the cervix. No pap smears were performed, and although specula were available in nine of the 18 study clinics, speculum examinations were not performed for any clients observed. Providers also reported that although they were trained that a urinalysis is a requirement for all new antenatal clients "... we cannot do even the simple one of litmus because we have no uri-sticks." 


\section{Figure 7 Percentage of antenatal clients asked about symptoms or examined for clinical signs of reproductive tract problems at pilot and standard clinics}

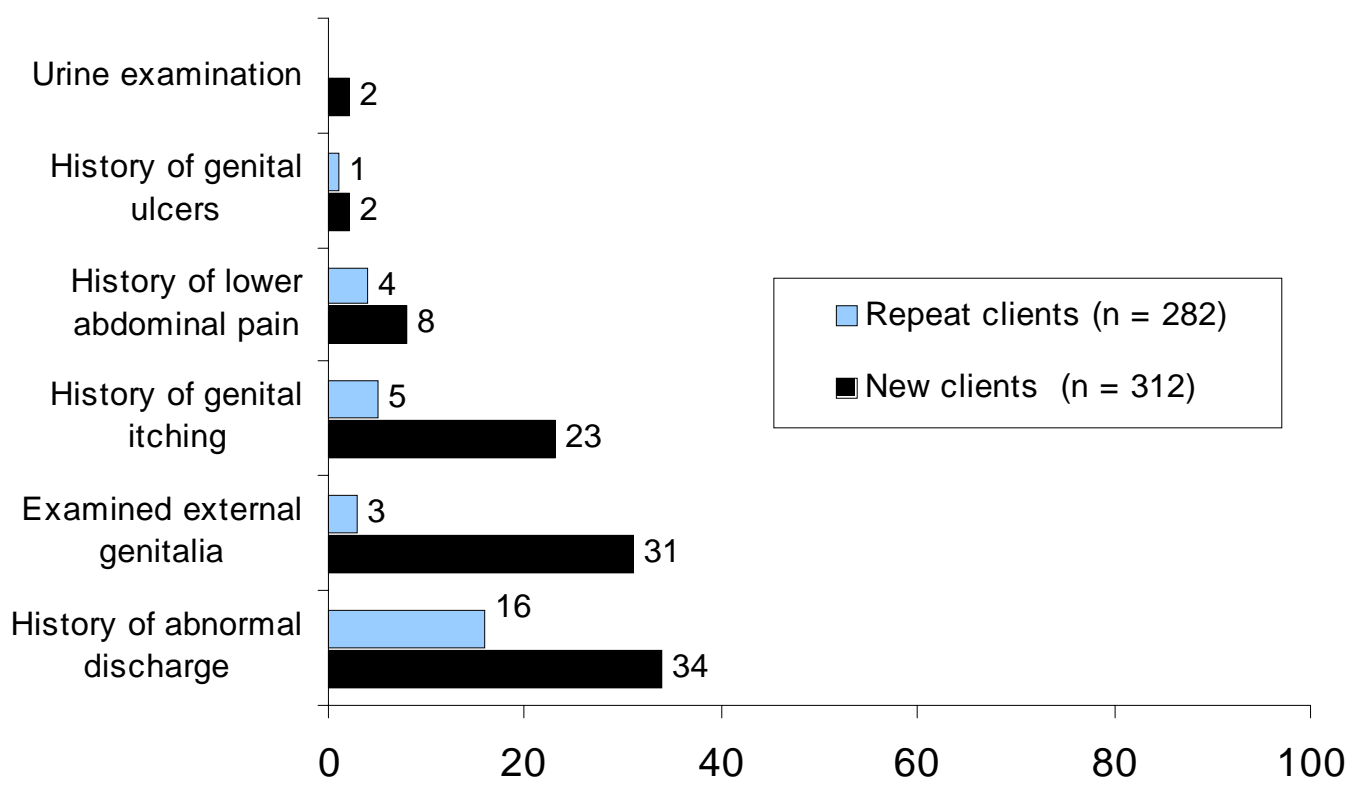

\section{Management Of Babies}

According to the training package used to train staff at the pilot clinics, all babies to mothers who were not treated during pregnancy, or who were treated late in pregnancy, or in the absence of information to confirm adequate treatment, should be treated. One of the following treatment regimes should be used: Procaine Penicillin G 50,000 units $/ \mathrm{kg} /$ day by intramuscular

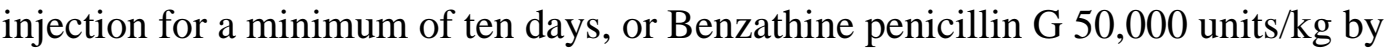
intramuscular injection as a single dose.

Management of babies born was not part of this case study. However a recent study undertaken at the main maternity hospital in Nairobi (Pumwani) among 22,466 women giving birth over the period April 1997 - April 1998 with a syphilis prevalence rate of three percent (Temmerman et al, 2000) found the incidence of adverse obstetric outcomes (low birth weight and stillbirth) to be 9.5 percent, with infected women having a four-fold higher risk of an adverse outcome than uninfected women. Those women who had been treated for syphilis during pregnancy still had a higher likelihood of an adverse outcome, but the odds were reduced to 2.5 times that of uninfected mothers. This study confirms the importance of the antenatal syphilis-screening programme as a means of improving pregnancy outcomes.

\section{Supervision And Monitoring}

Continuous supervision and monitoring are thought to have contributed to the pilot project's success (Jenniskens et al, 1995), in that it maintained staff motivation and morale, and helped to ensure that equipment and supplies were always present. According to the Medical Officer of Health for the NCC: "There is day to day supervision by sisters-in-charge at clinics and there is periodic supervision from the divisional heads at least once a month depending on the need and they give me feedback." The other NCC supervisors also said that they are supposed to visit the clinics at least once every three months, or preferably once a month. As 
one informant put it: "We are supposed to go every three months to the clinics to check on the quality of delivery of services, attitude of staff and even to talk to the clients."

However, when asked how many supervisory visits they had made in the last month, all six supervisors reported that they had not visited any of the clinics. The service providers, who also mentioned that the supervisors only visit when there is a problem and only when called upon by the sisters-in-charge, confirmed this. As one provider said: "they came when the gate was broken". "Yes they came to see the leaking roof," said another. In-depth interviews with the sisters-in-charge of clinics also confirmed this finding; more than one-third of them had not received any supervisory visit in the past six months. The providers said that the lack of regular supervision has demoralized them. As one provider put it: "Even the people who are sitting there at the head office do not visit us to know our problem. They do not know what we go through."

According to the key informants, factors affecting supervision include a lack of transport and fuel, telecommunications breakdowns and the shortage of supervisors. A lack of vehicles and fuel has crippled supervision of health facilities generally. For example, at the Special Treatment Clinic (STC), the only vehicle had been grounded for two weeks, and so supervisors could not deliver any supplies, provide quality control checks, or collect records. The lack of telephones has also affected supervision. One informant reported: "You know communication is absent ...it ceased in the 1990's and it died out between 1995-1996. So to communicate to the health centres even for monitoring purposes is difficult." In the absence of functioning telephones all supervision has to be through visits to the clinics but this is also not possible given the transport problems.

Irrespective of these problems, one key informant stated that "from my own knowledge of the Council, supervision is not one of the strongest aspects at the NCC. I have witnessed this since I joined the Council." Clearly the quality and quantity of supervision has greatly reduced since the pilot project was started in 1993.

\section{Time Utilization By Clients And Providers At The Clinics}

Providers indicated that in addition to the lack of equipment and supplies, there is often insufficient time to fully assess, counsel and test clients. This, they say, is for two reasons: the heavy client loads, and the fact that clients are in a hurry and get offended if they are delayed at the clinic.

NCC clinics are expected to be open to the public from 7.30am to 4.30pm. The Patient Flow Analysis (PFA) found that although clinics tended to open on time and clients arrived at the clinics before 7.30am, the first clients were usually not attended to until 9.00am. On average clients had to wait for about one hour before being attended to (45minutes for all clients, 50 minutes for new clients and 40 minutes for repeat clients).

"The nurse reports at work at 8.00 a.m. she stops to talk at the gate and 30 minutes are gone. She goes to change into uniform because some come with home clothes another 20 minutes are spent in dressing and talking. At 10.00 a.m. she goes for tea. Now how much has she done? The desire for the job is not there." "I always ask my nurses, you left your house with a purpose - to come and attend to these women at the clinic yet you are all over, then you have no business being here." (A supervisor at one of the study clinics) 
The PFA also found that clients are seen by up to five different providers in a single clinic visit for antenatal care. Much time is wasted between seeing the different providers. New antenatal clients waited for much longer between consultations than repeat clients (68 and 26 minutes respectively).

As would be expected, new antenatal clients who had blood specimens taken for syphilis testing spent more time at the clinic than other clients (Figure 8) - almost three hours to get a consultation that lasted an average of 31 minutes. The new antenatal clients who did not have their blood taken for the RPR test spent on average 15 minutes in direct contact with providers, suggesting that the procedure of drawing blood adds 16 minutes to the consultation time.

During exit interviews, more than three-quarters of the clients said that they were satisfied with the duration of the consultation and that the waiting time was reasonable. If this is true, the time available while clients wait could possibly be used for educational activities without inconveniencing the clients or drawing time away from the consultations.

Although a formal time motion study was not carried out, information from the PFA and service statistics from the clinics suggest that staff at the pilot clinics spend 37 percent of their paid working time in direct contact with the clients, while staff at the standard clinics spent 19 percent of their time in contact with clients. In the absence of a time motion study, it is not possible to determine how much of the remaining portion of staff time is spent on other clinic activities and how much is spare time that could be used for improving on quality of services provided.

\section{Figure 8 Time utilization at the clinic}

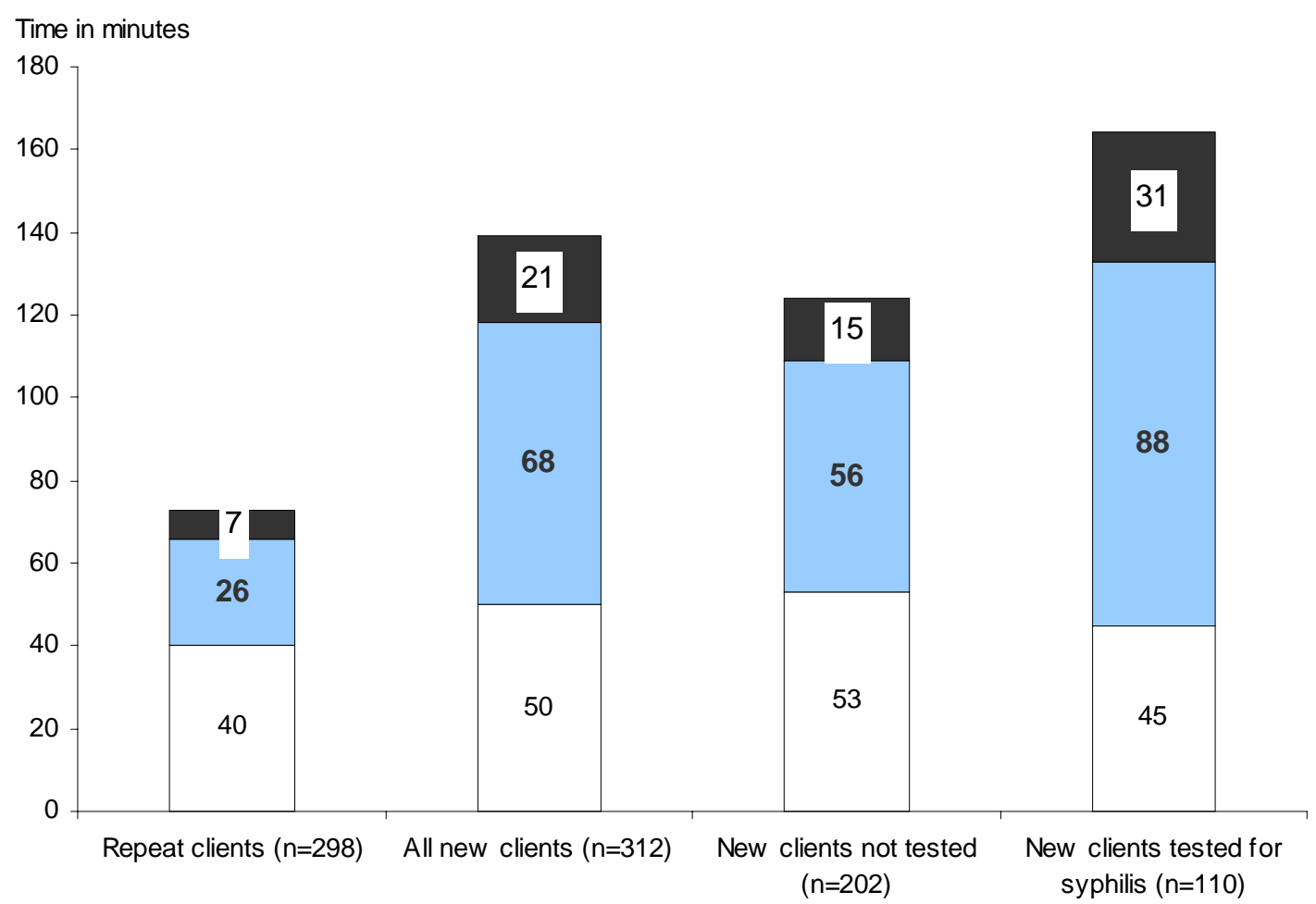




\section{PROGRAMME PERFORMANCE}

Service statistics for a continuous period of 12 months preceding the study were reviewed for all the study clinics. However, complete data for a continuous period of twelve months preceding the study were available for the indictors of interest for only nine of the eleven pilot clinics, and for three of the seven standard clinics, and so only data from these clinics is reported (see Appendix 1 for a listing of clinics included in this performance analysis).

\section{Proportion Of Antenatal Clients Screened For Syphilis}

The nine pilot clinics served 22,886 new antenatal clients in the twelve months preceding the study, i.e. approximately 2,500 clients per clinic. According to the clinic records, 81 percent of new antenatal clients were tested for syphilis. These findings indicate that although the majority of antenatal women continue being screened, and the proportion is still higher than with the standard referral approach, there has been a significant reduction since 1993 when nearly 100 percent of women were tested (Figure 9). The proportion of clients being screened off-site (i.e. in clinics before the pilot project and currently in the standard clinics) has remained fairly constant since the early 1990s at between 50-60 percent.

Figure 9: Proportion of antenatal clients screened for syphilis

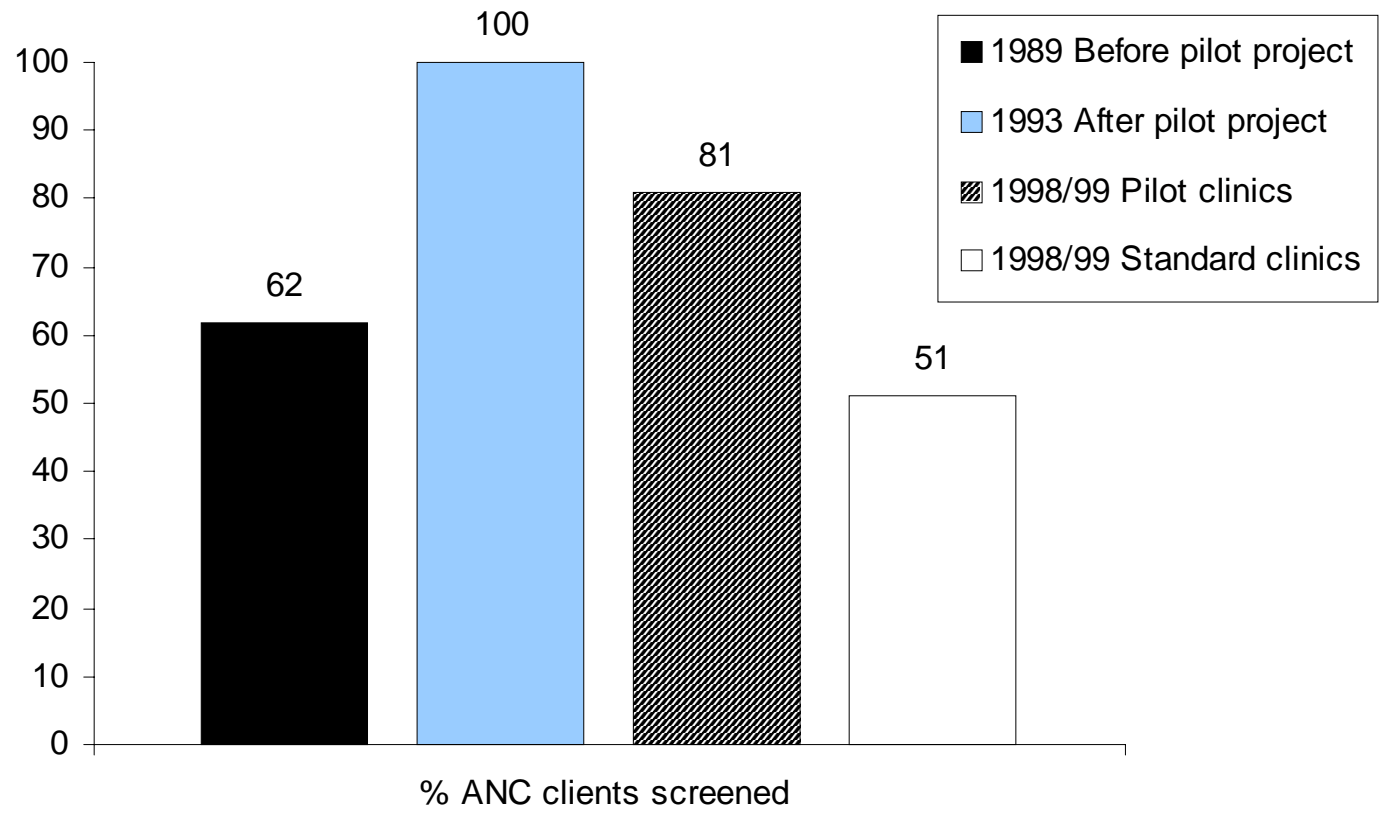

When interviewed, programme managers attribute this drop to the frequent shortages of supplies for taking blood and for performing the test at these clinics. As described earlier, observations of a sample of new antenatal clients showed an even lower proportion (40\%) being tested. The fact that some antenatal clients are tested on later visits because of a lack of equipment or supplies may partially explain this apparent discrepancy between the proportion observed being tested during the case study and the proportion recorded in clinic records as having been tested. 
This discrepancy may also be due to other reasons. A possible explanation is a difference in programme performance between when the service statistics were recorded (April 1998 April 1999) and when the case study was conducted (March-April 2000). To explore this possibility, clinic records from the pilot clinics for the period January - June 2000 were also examined. Over this period a total of 14,054 new antenatal clients were attended at the nine pilot clinics, of which 8,332 (60\%) were tested for syphilis. Although the proportion tested is lower than that reported for the period in 1998/1999, it is higher than the proportion observed during the two days of the case study.

This suggests that observations over a short period of time might underestimate the true proportion of women who get tested during antenatal care, and may be a function of the irregular supply of testing reagents and supplies. For example, over this six months period, on average each of the clinics was able to carry out syphilis tests for only 34 out of the 120 days that the clinics were open because of such shortages. Whatever the reason, however, these data also support the suggestion that the proportion of women screened is decreasing over time.

Of the women who were tested during the period 1998/1999, 534 (3.2 percent) were found to be positive for syphilis infection. This confirms a steady decline in antenatal syphilis prevalence from 7.3 percent in 1995, which has reversed a previous increase observed over the 1990-1994 period (Temmerman et al, 1999). These data give a strong indication that the decentralised antenatal syphilis-screening programme is having a positive effect on reducing syphilis prevalence in Nairobi.

\section{Proportion Of Antenatal Clients Testing Positive That Are Treated}

The proportion of those women testing positive that are actually treated was increased tremendously by the introduction of the decentralised model - from nine percent to 87 percent. As can be seen in Figure 10, the NCC has been able to sustain this level of treatment in the pilot clinics and increase it further to 95 percent.

Moreover, it has also managed to ensure that clients attending the standard clinics, although less likely to be screened, are equally likely to be treated if found positive, a finding that supports the results of another study undertaken in 1997/1998 (Temmerman et al, 2000). A possible explanation of the similarity between the proportion of clients treated at both the pilot and standard clinics is that when clients are referred to the pilot clinics for testing they can also get treated there. However, the source of treatment is not indicated in the clients' record cards thus it is not possible to determine the proportion of clients at the standard clinics who received treatment elsewhere. 
Figure 10: Proportion of antenatal clients testing positive who were treated

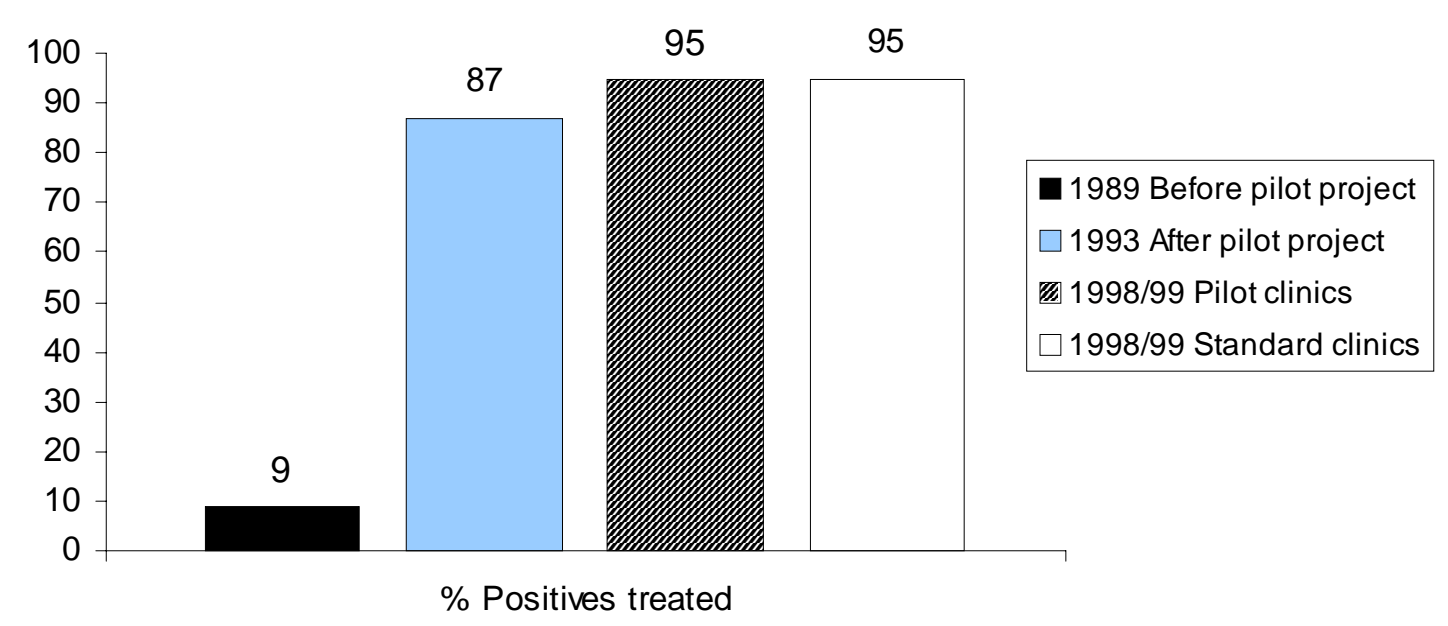

\section{Proportion Of Partners Treated}

There are no records of the number of clients testing positive who were given a partner notification slip or who were verbally requested to ask their partners to come for treatment. However, service statistics based on clinic records over the previous twelve months for the nine pilot clinics indicated that 70 percent of the partners of clients who tested positive returned to the same facility and were treated. There was no comparable data from the standard clinics, making it difficult to attribute this high rate of partner treatment to the decentralization of testing and treatment of maternal syphilis. However, this rate is much higher than the 50 percent reported during the pilot programme (Jenniskens et al, 1995), suggesting that this component has subsequently improved even further.

These findings suggest that when efforts are made the partners will come for treatment. Moreover, two-thirds of the clients interviewed said that they thought their partners would accompany them to the clinic if they were requested to do so. A key factor to this success is probably because the partner is invited to come to the clinic without disclosing the reasons for the invitation. The high response rate reflects the overall concern that partners attach to pregnancy and pregnancy outcome.

A study undertaken in 1997 - 1998 on partner notification among pregnant women at Nairobi's Pumwani Maternity Hospital (at which most women attending NCC clinics will deliver) found a similar level of partner notification and treatment (67\%) among pregnant women diagnosed as having syphilis (Gichangi et al, 2000). Interestingly, Gichangi et al found also that the rate of partner notification and treatment among women found to have syphilis after delivery was only 36 percent. This reinforces the conclusion that partner notification for antenatal syphilis can be effective because of the concern for the unborn baby, whereas the effectiveness drops off postpartum.

Gichangi et al propose that improved counselling of the women during screening about the importance of partner notification and educational messages to the general community may enhance partner referral, but they remain sceptical about the cost-effectiveness of active tracing by trained staff ('provider referral'). Gichangi's study also found a sizeable (7\% vs. 
$19 \%$ ), although not statistically significant, reduction in the proportion of pregnancies with adverse obstetrical outcomes (low birth weight and stillbirth) among syphilis-infected women whose partners had been treated compared with those among infected women whose partners were not treated.

\section{Programme Costs}

The programme costs of antenatal visits, including the costs of screening and testing blood for syphilis, and the costs of treating syphilis during antenatal visits were calculated. Information on the supplies and their costs used to collect blood, as well as the laboratory costs to test the blood for syphilis, was obtained through interviews with programme managers and service providers and quotations from suppliers. In addition, information on other supplies used for antenatal visits that were not specifically related to drawing blood (such as costs for collecting urine and giving tetanus injections) was also collected using the same sources. Data on the salaries and benefits of the personnel who either provided services to the women making the antenatal visits or supervised the staff who provided these services at the six pilot clinics was obtained from the personnel department at the NCC. The following costs were calculated using this information.

Direct labour costs: included only the cost of the time that providers spent with clients.

Indirect labour costs: included the time that providers did not spend with clients but for which they were paid. The cost of support staff time (which included the proportions of time that the nurse aids, clerks, sister-in-charge and the doctor contributed to the provision of antenatal care) was calculated separately.

Capital equipment and clinic infrastructure costs: these were calculated using replacement costs supplied by the NCC. A useful life of five years was assumed for each piece of equipment and a discount rate of 5 per cent per annum was used to discount the costs. The cost of infrastructure was calculated by estimating the clinic space that is dedicated to antenatal care and the current rents for office space in Nairobi.

Support staff time: These were calculated using information provided by the $\mathrm{MOH}$ in-charge of the NCC services regarding the staffing situation at each clinic. At the time of the study, each of the NCC health service provision zones had at least one doctor who spent about 10\% of their time supporting ANC services at the clinics. Each of the clinics had one sister incharge of the ANC services, one cleaner and a clerk responsible for the client records.

Clinic supplies: these were calculated by first determining the quantity required per client visit through interviews with the service providers, and then multiplying this by the unit cost provided by the supplies office of the NCC. Although no treatment was observed, the cost of drugs and supplies required to treat syphilis infection as recommended by WHO and the $\mathrm{MOH}$ were estimated using the prevalence rates estimated from clinic service statistics.

These costs were calculated for the different types of visits (new antenatal client not tested, new antenatal client tested, revisit client) and for the pilot and standard clinics. The average costs for revisit clients were US\$2.60 at the pilot clinics and US\$3.80 at the standard clinics (1US\$ $=$ KShs 80 at the time of the study).

Figure 11 shows that, on average, it costs the NCC US $\$ 5.40$ and US\$6.60 per client to provide antenatal care services without undertaking screening and treatment for maternal syphilis infection at the pilot and standard clinics respectively. Providing antenatal care by 
using the standard referral approach to screen and manage maternal syphilis infection increases the cost to US\$11.30 per new client.

\section{Figure 11 Costs per visit by type of client management protocol}

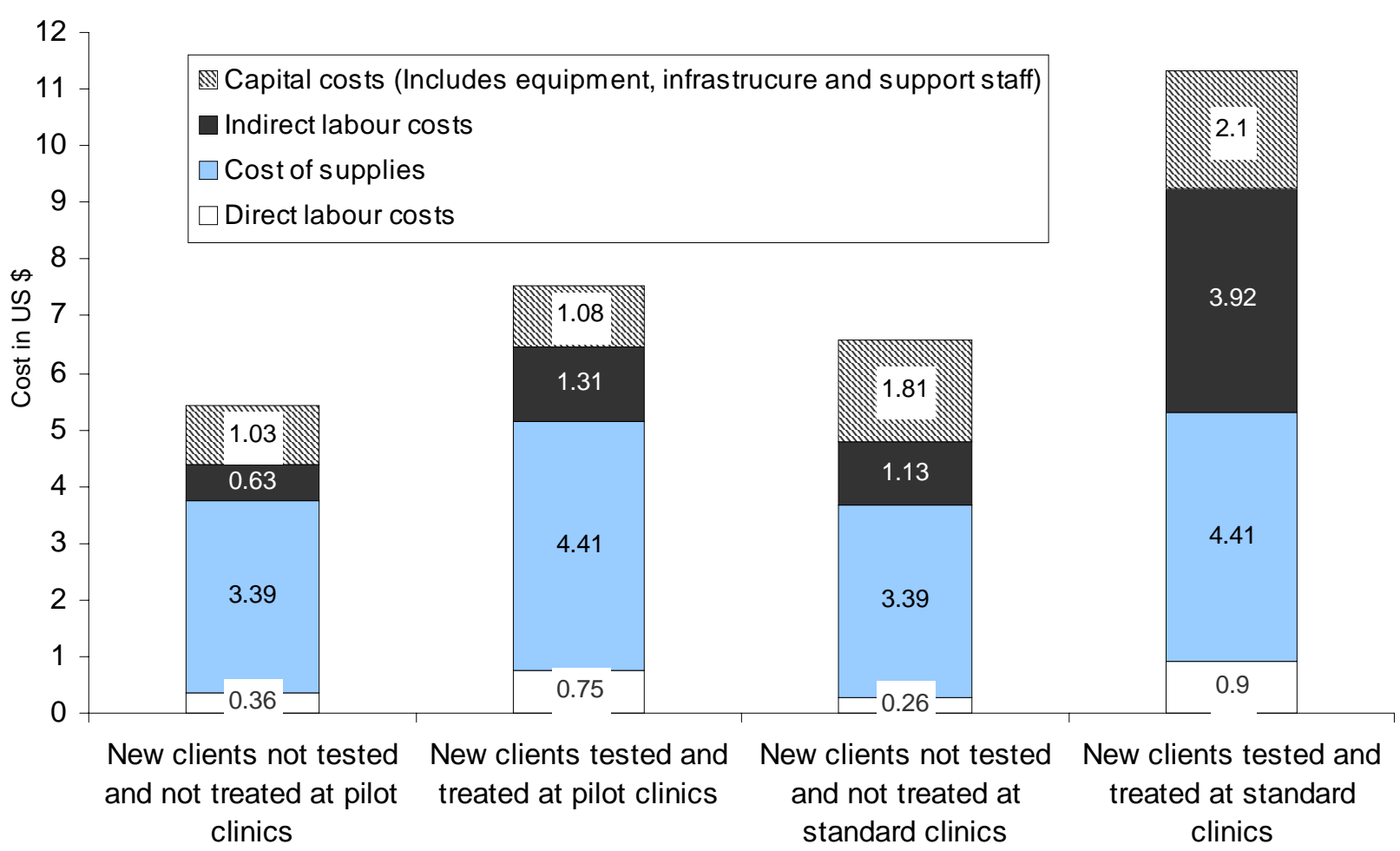

On the other hand, providing antenatal care and syphilis screening using the decentralised approach costs US\$7.60 per new client. Thus, introducing the decentralized approach to maternal syphilis screening and management at the standard clinics would increase the pervisit cost from US\$6.60 to US\$7.60, a difference of US\$1.00.

For both types of visit the average costs are higher in the standard clinics than in the pilot clinics. The major difference between the two types of clinics is in the costs associated with indirect labour and capital equipment. This is not surprising, given that these two costs are inversely related to the client loads served by the clinics, and the pilot clinics had larger clients loads compared with the standard clinics.

The costs for medical supplies for general antenatal care and for syphilis screening and treatment are assumed to be the same for both types of clinics. This is because the standard clinics refer their clients for testing at the pilot clinics where similar cadres of personnel using the same type of equipment and reagents carry out the tests.

The total average annual cost per clinic using the above estimates and taking into account all types of visits was calculated for three different approaches to the provision of antenatal care. If the NCC programme were to provide only antenatal care without syphilis screening and treatment, it would cost US $\$ 31,567$ per year for each of the pilot clinics and US $\$ 23,885$ per standard clinic. The cost per clinic when the programme adds syphilis screening and treatment increases by US $\$ 4,545$ for the pilot clinics compared to US $\$ 2,925$ for the standard 
clinics. The apparent large increase in cost for the pilot clinics compared to the standard clinics is a reflection of the higher volume of clients attended to at the pilot clinics.

Considering the overall per clinic costs alone suggests that the standard referral approach is cheaper than the decentralized approach. However, the differences in total costs are directly related to the number of clients served, and as the pilot clinics serve more clients the cost per clinic is higher, although the cost per client served is lower. 


\section{CONCLUSIONS}

This case study has assessed the readiness of the NCC's antenatal care programme to offer a decentralized syphilis screening and treatment service, the quality of service provided, and the effectiveness and cost of offering such a service. More detailed information was collected that the NCC can use to strengthen the programme, and these results are given in the main body of the report. The key findings, and those that will be of interest to other health programmes in Kenya and the region, are as follows.

- A decentralized model of on-site syphilis screening using the RPR test, treatment with a single dose of penicillin, and pro-active partner notification is feasible to implement within an existing antenatal programme, and enables the client to have a "one-stop", same-day service.

- The average cost per new antenatal client visit with syphilis screening and treatment was only slightly higher $(\$ 1.00)$ at the pilot clinics than the cost of an antenatal visit without syphilis screening and treatment at the standard clinics. The cost per antenatal client screened and treated at the pilot clinics is only two-thirds of the cost per antenatal client screened and treated at the standard clinics.

- On-site treatment, whether associated with on-site testing or referral testing, is much more effective for successfully managing infected women than referring infected women elsewhere for treatment.

- Pro-active partner notification, through counselling women on its importance and using contact slips to inform the partner of the need for treatment, can reach and treat at least two-thirds of partners.

- Antenatal clients spend a lot of time waiting to be attended at the clinics. Several different providers attend each antenatal client in a single visit a practice that further increases the waiting time for clients. Of the total time spent at the clinics a very small portion is utilized for consultations.

- Service providers in the NCC antenatal clinics with the decentralized approach spend 37 percent of their paid time attending to clients, compared to 19 percent of time spent by staff at the standard clinics.

- In common with most antenatal services in sub-Saharan Africa, the NCC clinics are insufficiently prepared at present to offer syphilis screening and treatment to all antenatal clients. This is not because of a lack of testing facilities, but because of a lack of basic supplies of needles and syringes for collecting blood and delivering treatment, and of the treatment drugs themselves.

- The effectiveness and quality of the service offered could also be improved through ensuring that all nurses in antenatal clinics have at least basic training in syphilis screening and management. This skill should be developed within the context of integrating broader STI and HIV/AIDS prevention and management into antenatal care. 
This case study did not set out to determine the impact of the programme on syphilis prevalence among pregnant women or in the general population. However, according to the service statistics reviewed, the proportion of women screened who tested positive decreased from 6.5 per cent in 1993 to 3.2 per cent in 1999/2000, indicating that there may have been an impact.

Finally, it is important to note that this model was introduced at a time when the most rapid test available for syphilis was the RPR test. Since then, tests for syphilis that do not require equipment or supplies have been developed. Consequently, it is now even easier and less costly for health programmes to introduce on-site syphilis testing, not only for antenatal clients but also for the general population. Programmes that currently do not offer such services should be encouraged to do so, especially those located in populations with a high prevalence of syphilis. 


\section{REFERENCES}

Gichangi, P., K. Fonk, C. Sekadde-Kigondu, J. Ndinya-Achola, J. Bwayo, P. Claeys and M. Temmerman. 2000. Partner Notification of pregnant women infected with syphilis in Nairobi, Kenya. Int. J of STD and AIDS, 11:257-261.

Jenniskens F., E. Obwaka, S. Kirisuah, S. Moses, F. Yusufali, J. Ndinya-Achola, L. Fransen, M. Laga, and M. Temmerman. 1995. Syphilis control in pregnancy: decentralization of screening facilities to primary care level, a demonstration project in Nairobi, Kenya. Int J Obstet Gynecol, 48:S121-8.

Kenya Demographic and Health Surveys KDHS. 1998. Kenya Demographic and Health Survey, Macro International Inc. Calverton, Maryland, USA.

Maggwa B.N., I. Askew, C. Marangwanda, et al. 1999. Demand for and cost-effectiveness of integrating RTI/HIV services with Clinic-based family planning services in Zimbabwe. Africa OR/TA II Project, Population Council, Nairobi, Kenya.

Solo J., B.N. Maggwa, K. J. Wabaru, et al. 1998. Improving the management of STIs among MCH/FP clients at the Nakuru Municipal Council Health Clinics. Africa OR/TA II Project, Population Council, Nairobi, Kenya.

Temmerman, M., M. I. Lopita, H. C. G. Sanghvi, et al. 1992. The role of maternal syphilis, gonorrhoea and HIV-1 infections in spontaneous abortion. Int. J. of STD and AIDS, 1992; 3: 418-422.

Temmerman, M., F. Mohamedali and L. Fransen. 1993. Syphilis prevention in pregnancy: an opportunity to improve reproductive health and child health in Kenya. Health Policy and Planning. 1993; 8(2): 122-127.

Temmerman, M., Fonk, K., Bashir, F., et al. 1999. Declining syphilis prevalence in pregnant women in Nairobi since 1995: another success story in the STD filed? Int. J. STD and AIDS. 1999; 10: 405-408.

Temmerman, M., P. Gichangi, K. Fonk, et al. 2000. Effect of syphilis control programme on pregnancy outcome in Nairobi, Kenya. Sex. Trans. Infect; 76(2): 117-121.

van Dam, C.J. 1995. HIV, STD and their current impact on reproductive health: the need for control of sexually transmitted diseases. Int. J. Gyn. and Obs. 50 Suppl. 2(1995) S121S129.

Welsh, M., P. Feldblum and S. Chen. 1997. Sexually transmitted disease risk assessment used among low-risk populations in East/Central Africa: A review. East Afr. Med J. 1997; 74(12):764-771. 


\section{APPENDIX 1}

\section{NCC CLINICS INCLUDED IN THE CASE STUDY}

\section{Decentralised Pilot Clinics}

Umoja

Makandara

Kangemi

Kariobangi

Mathare North

Baba Dogo

Langata

Westlands*

Eastleigh*

Riruta

Dandora

\section{Standard clinics}

Kayole

Karen

Mathare Lions

Waithaka

Pumwani

Charles New

Bahati

* Clinics not included in the performance analysis 